\title{
Complement-Dependent Synaptic Uptake and Cognitive Decline after Stroke and Reperfusion Therapy
}

\author{
${ }^{-}$Ali M. Alawieh, ${ }^{1,2}$ E. Farris Langley, ${ }^{2}$ Wuwei Feng, ${ }^{3}$ Alejandro M. Spiotta, ${ }^{4}$ and ${ }^{\circledR}$ Stephen Tomlinson ${ }^{2,5}$ \\ ${ }^{1}$ Department of Neurosurgery, Emory University School of Medicine, Atlanta, Georgia 30322, ${ }^{2}$ Department of Microbiology and Immunology, \\ Medical University of South Carolina, Charleston, South Carolina 29425, ${ }^{3}$ Department of Neurology, Duke University Medical Center, Durham, NC, \\ 27710, ${ }^{4}$ Department of Neurosurgery, Medical University of South Carolina, Charleston, South Carolina 29425, and ${ }^{5}$ Ralph H. Johnson VA Medical \\ Center, Charleston, South Carolina 29401
}

Despite the success of reperfusion therapy in significantly reducing the extent of infarct expansion after stroke, the effect of revascularization on poststroke neuroinflammation and the role of anti-inflammatory strategies in postreperfusion era are yet to be explored. Here, we investigate whether the neuroinflammatory response may still contribute to neurologic deficits after reperfused stroke by using targeted complement inhibition to suppress poststroke neuroinflammation in mice with or without concurrent reperfusion therapy. Complement inhibition was achieved using B4Crry, an injury site-targeted inhibitor of C3 activation. Following embolic stroke in male $\mathrm{C} 57 \mathrm{bl} / 6$ mice, thrombolysis using tissue-plasminogen activator ( $\mathrm{t}-\mathrm{PA}$ ) reduced injury and improved motor deficits, but did not improve cognitive outcomes. After both reperfused and non-reperfused stroke, complement activation and opsonization of hippocampal synapses directed ongoing microglia-dependent phagocytosis of synapses for at least $30 \mathrm{~d}$ after stroke, leading to a loss of synaptic density that was associated with cognitive decline. B4Crry treatment, alone or in combination with IPA, limited perilesional complement deposition, reduced microgliosis and synaptic uptake, and improved cognitive outcome without affecting regenerative responses. Furthermore, complement inhibition improved the safety, efficacy, and treatment window of reperfusion therapy with t-PA by limiting hemorrhagic transformation. This work thus demonstrates that poststroke neuroinflammation contributes to hemorrhagic transformation and progression of neurodegenerative responses in the brain even following early and successful revascularization.

Key words: cognitive outcomes; complement; reperfusion; stroke; tissue-plasminogen activator

Significance Statement

This study addresses two major challenges facing the treatment of stroke in the era of reperfusion therapy: hemorrhagic transformation and the disconnect between successful revascularization and functional outcomes. We studied how complement-dependent neuroinflammation drives the pathophysiology behind these challenges using a translationally relevant strategy. Complement inhibition was achieved using B4Crry, an injury site-targeted inhibitor of C3 activation. Following embolic stroke, pharmacological thrombolysis limited infarct size, but did not prevent complement activation. In reperfused and nonreperfused stroke, complement activation and opsonization of hippocampal synapses resulted in synaptic phagocytosis and subsequent cognitive decline. B4Crry treatment limited perilesional complement deposition, reduced microgliosis and synaptic uptake, and improved cognitive outcomes. Complement inhibition also improved the safety, efficacy, and treatment window of thrombolytic therapy.

\section{Introduction}

Revascularization therapy, both pharmacological and mechanical, is the standard of care for acute ischemic stroke (AIS; Hacke et al., 2008; Goyal et al., 2016; Powers et al., 2019). Although advances in endovascular interventions have led to significant improvements in AIS outcomes, it is estimated that $<15 \%$ of stroke patients are likely eligible for revascularization therapy. In

S.T. is an inventor on a patent application for natural antibody-targeted complement inhibitors filed by the University of Colorado (PCT/US2014/012831; targeting constructs based on natural antibodies and uses thereof) and is a consultant for Q32 Bio, a company developing complement inhibitors. All other authors declare no competing financial interests.

Correspondence should be addressed to Stephen Tomlinson at tomlinss@musc.edu or Ali M. Alawieh at aalawie@emory.edu.

https://doi.org/10.1523/JNEUROSCI.2462-19.2020

Copyright $\odot 2020$ the authors 
addition, of patients who meet criteria for intervention, $<50 \%$ have functional independence at $90 \mathrm{~d}$, despite $>80 \%$ rate of successful vessel recanalization (Goyal et al., 2016; Babadjouni et al., 2017), and at least $10 \%$ of patients develop poststroke dementia (Chen et al., 2013). One major reason for limited recovery is that beyond vessel occlusion, cerebral ischemia and reperfusion unleashes a complex physiological response that involves robust neuroinflammatory and neurodegenerative processes that are not targeted by revascularization therapy (Alawieh et al., 2018b, c). In fact, preclinical studies have shown that while reperfusion therapy alone is effective at reducing infarct volumes, cerebral reperfusion after the initial ischemic insult is associated with persistent and ongoing perilesional neuroinflammation (Iadecola and Anrather, 2011). Thus, adjuvant anti-inflammatory neuroprotective interventions that target alternate pathophysiological mechanisms may mitigate the disparity between recanalization success rates and motor and cognitive recovery in patients. Nevertheless, targeting the postischemic inflammatory response has been a major challenge due to the complexity of the response involving multiple and diverse mediators.

In this work, we used a murine microembolic model (MicroE) of IS which presents a clinically relevant paradigm to investigate stroke in the context of reperfusion therapy (Alawieh et al., 2016). Early administration of tissue-plasminogen activator ( $t-$ PA) following embolization of the middle cerebral artery (MCA) results in rapid reperfusion and minimal infarct and motor deficits. Therefore, this allows for optimal investigation of the effect of the neuroinflammatory response in the brain independent of infarct size by studying efficacy of interventions in reperfused animals. The majority of neuroprotective and anti-inflammatory agents have been tested for efficacy in models that involve large infarcts and the independent contribution of postischemic neuroinflammation is difficult to disentangle. Another limitation of current approaches in neuroinflammation is the absence of targeted strategies (Alawieh and Tomlinson, 2016). We recently developed an approach that limits the postischemic inflammatory response locally in the penumbra by using an injury site-targeted complement inhibitor (Alawieh et al., 2018b). Activation of the complement system is an upstream event of the inflammatory cascades, and it represents a promising target for acute stroke therapy, since its inhibition will impact the generation of multiple downstream mediators. This site-targeted therapeutic strategy involves linking the complement inhibitor, Crry, to a single chain antibody $(\mathrm{scFv})$ that recognizes a danger associated molecular pattern expressed on Annexin IV (Kulik et al., 2009). The epitope recognized by B4scFv is injury specific and is expressed specifically in the postischemic penumbra following experimental and human stroke (Alawieh et al., 2018b). The targeted complement inhibitor, termed B4Crry, inhibits all complement pathways at the central C3 activation step, and thus inhibits the generation of $\mathrm{C} 3$ opsonins and $\mathrm{C} 3 \mathrm{a}$, as well as the downstream products $\mathrm{C} 5 \mathrm{a}$ and the membrane attack complex.

In this work, we use the combination of reperfusion therapy with thrombolysis and complement inhibition with B4Crry to investigate whether the neuroinflammatory response persists after thrombolysis and suppression of infarct volume and whether there is still opportunity for neuroprotective therapy even in the context of reperfusion therapy. This work is the first to demonstrate that despite rapid reperfusion with significant reduction in acute infarct and normalization of motor deficits after thrombolytic stroke therapy in mice, complement-initiated neuroinflammatory responses are still triggered acutely and contribute to chronic inflammation. This chronic response resulted in complement-dependent synaptic degeneration and poor cognitive recovery, even in the context of rehabilitative interventions. Inhibition of complement using B4Crry improved the safety and efficacy of thrombolytic therapy and inhibited complement-dependent neurodegeneration leading to improved chronic cognitive outcomes. Supporting our data in murine models, we also investigated the pattern of motor and cognitive recovery in human stroke patients treated with reperfusion therapy.

\section{Materials and Methods}

\section{Study design}

For all studies involving behavioral assessment (cognitive and motor), animals were acclimated on behavioral tasks during the week before stroke induction. Animals were subjected to stroke using either the intraluminal filament occlusion of the MCA (MCAO) model or the microembolic model (MicroE) of MCAO. For acute studies, animals were euthanized at 1 or $3 \mathrm{~d}$ after stroke. For chronic studies, animals were followed for up to $30 \mathrm{~d}$. Before stroke surgeries, animals were randomized into different treatment groups using an automated script. Animals were tested for motor and cognitive recovery at different time points after stroke, and brains were collected at predetermined endpoints for histologic and molecular analyses. Lab personnel involved in surgery and testing of animals were blinded to the group allocation, and personnel performing the pharmacological injections were blinded to the group allocations. Exclusion criteria for animals were: failure to achieve $>70 \%$ reduction of cerebral blood flow (CBF) on Doppler scanning, intraoperative mortality, or mortality before recovery from anesthesia and receiving vehicle, t-PA, or B4Crry treatment. Animals excluded due to failed CBF reduction or peri-operative mortality were not included in mortality rates calculated for each group. Statistical analyses were performed with the last observation carried forward for animals that died after day 1 of stroke. Analysis of behavioral data were performed using automated video-analysis tools whenever possible, to avoid observer bias. Unbiased stereology was used in field selection for histologic analyses, and data were quantified using automated macros. Sample size was estimated before initiation of the studies based on prior data using B4Crry in stroke models, and was based on a power $>80 \%$, and an $\alpha<5 \%$ that is corrected for multiple comparisons whenever needed.

\section{Recombinant proteins}

B4 IgM mAb (anti-Annexin IV; Kulik et al., 2009) was expressed in a hybridoma cell line (kindly provided by V. M. Holers, University of Colorado) as previously described (Atkinson et al., 2015; Alawieh et al., 2016). B4scFv derived from the $\mathrm{B} 4 \mathrm{mAb}$ hybridoma, and the $\mathrm{B} 4 \mathrm{scFv}$ Crry (B4Crry) fusion protein were prepared as previously described (Atkinson et al., 2015; Alawieh et al., 2016). Proteins were purified in an endotoxin-free environment and concentrated in PBS before administration to experimental animals.

\section{Animal husbandry and care}

The Institutional Animal Care and Use Committee (IACUC) at the Medical University of South Carolina approved all experiments involving vertebrate animals. Wild-type (WT) C57BL/6j mice were obtained from The Jackson Laboratory and housed in Helicobacter negative rooms at the Medical University of South Carolina (MUSC) animal facility. Animals were housed as a group of four littermates per cage with corn cob bedding and paper nestlings and were maintained in their home cages unless assigned to rehabilitation cages. Cages were changed every $10-14 \mathrm{~d}$ and were cleaned and autoclaved. Mice received regular chow food and water ad libitum with the addition of DietGel Recovery (clear $\mathrm{H}_{2} \mathrm{O}$ ) in the immediate postoperative period. Light/dark cycles were set at 12/12 h switching at 6 A.M. and 6 P.M. Behavioral testing was performed during the day (light phase). Testing was performed between 9 A.M. and 2 P.M. with fixed timing for each task. 


\section{Stroke surgeries}

Adult male C57BL/6 mice were allowed to acclimate in the animal facility for one week before use. Animals were 12 weeks old at the day of stroke induction or sham surgery. Three different stroke models were used: permanent MCAO (pMCAO) and transient MCAO (tMCAO) using intraluminal filament (Chiang et al., 2011) and a MicroE that we have previously described (Alawieh et al., 2016). The MCAO models have been described before (Chiang et al., 2011; Alawieh et al., 2016). To better investigate how the neuroinflammatory response triggered by complement inhibition interacts with reperfusion therapy by $\mathrm{t}$-PA, we used the MicroE (described in Alawieh et al., 2016). During surgery, physiological monitoring of heart rate and temperature was performed to ensure no differences across the groups. After surgery, animals were maintained on a heating pad with a target temperature of $37^{\circ} \mathrm{C}$ until recovery and they were monitored every $15 \mathrm{~min}$ for the first $6 \mathrm{~h}$ before transfer to housing facility. Soft food (DietGel Recovery, clear $\mathrm{H}_{2} \mathrm{O}$ ) was provided during the first $48 \mathrm{~h}$ after surgery.

\section{tMCAO stroke model}

Mice were anesthetized using ketamine $(80-100 \mathrm{mg} / \mathrm{kg}$, i.p.) and xylazine $(10 \mathrm{mg} / \mathrm{kg}$, i.p.). Following neck dissection, the right common carotid artery (CCA), external carotid artery (ECA), and internal carotid artery (ICA), were dissected and identified. A permanent silk tie was placed around the most distal end of the ECA, and a loose temporary suture placed around the ECA at the more proximal close to bifurcation. A temporary clip was placed around the CCA. A small incision was made in the ECA between the two sutures, and a custom-made monofilament suture with thick tip, sized to the animal's weight (RWD Inc.), was inserted into the ECA up to the origin of the ICA and then distally through the ICA to land at the origin of the MCA. Reduction in blood flow was confirmed via laser Doppler flowmetry. After $1 \mathrm{~h}$, the filament was withdrawn, the temporary suture tied to occlude the ECA, and the clip is removed from the CCA allowing for reperfusion.

\section{pMCAO stroke model}

This model is performed similar to the tMCAO model with the exception that the filament is permanently placed in position and not removed after ligation.

\section{MicroE stroke model}

Microemboli were prepared from human plasma as previously described in detail (Alawieh et al., 2016). The prepared clot was homogenized into small emboli as described (Alawieh et al., 2016) and sized to $2-4 \mu \mathrm{m}$ in diameter by repeated homogenization. Emboli were counted using a Z2 Coulter particle analyzer (Beckman Coulter) to ensure target concentration and confirm that $<5 \%$ of particles are above $10 \mu \mathrm{m}$ in diameter. To induce target vessel occlusion, we used a high dose of microemboli consisting of $1.5 \times 107$ emboli per animal, concentrated in $100-\mu$ PBS. Following emboli preparation, mice were anesthetized using ketamine $(80-100 \mathrm{mg} / \mathrm{kg}$, i.p.) and xylazine $(10 \mathrm{mg} / \mathrm{kg}$, i.p.), and a polyethylene catheter with inner diameter of $0.02 \mathrm{~mm}$ (BrainTree Scientific) loaded with high-dose emboli was inserted to the origin of the MCA via an incision through the ECA while clamping the CCA. Emboli were then injected at the origin of the MCA. Adequate vessel localization of the catheter was confirmed by Evans blue (EB) injection and assessment of involved vessel distribution. Reduction in blood flow was confirmed via laser Doppler flowmetry. Time of emboli administration was considered the time of ischemia induction.

\section{Animal treatment}

For the MicroE model, following emboli administration, mice were treated with PBS vehicle or human recombinant t-PA (Cathflo Activase, Alteplase, Genentech), either with or without B4Crry. Treatments were via tail vein injection at a dose of $10 \mathrm{mg} / \mathrm{kg}$ for t-PA and $16 \mathrm{mg} / \mathrm{kg}$ for B4Crry. Treatments were administered at 2, 4, or $6 \mathrm{~h}$ after emboli injection, and for co-treated animals, B4Crry was administered immediately after t-PA.

\section{C3a levels and hemoglobin content}

For molecular analyses of Hemoglobin content and C3a levels, acutely euthanized animals were decapitated and brains were extracted, and the ipsilateral hemisphere used for analysis. Following the protocol recommended by the manufacturer, C3a levels were measured using mouse C3a ELISA kit (Kamiya Biomedical). Nafamostat mesylate (FUT-175, Sigma-Aldrich) was used to inhibit further complement activation during preparation of homogenates. Hemoglobin content in brain homogenates was measured using Drabkin's reagent (D5941; Sigma-Aldrich) according to the manufacturer's recommendations (Alawieh et al., 2018c).

\section{Blood-brain barrier (BBB) integrity assessment}

To assess BBB integrity, we used EB fluorescence in brain homogenates as previously described (Uyama et al., 1988). In brief, $2 \% \mathrm{~EB}$ in saline was administered intravenously via tail vein injection to poststroke mice $2 \mathrm{~h}$ before killing. Following killing and intracardiac perfusion with 10$\mathrm{ml}$ PBS, the ipsilateral hemisphere was homogenized and EB fluorescence measured by fluorescent spectroscopy using appropriate external standards (Uyama et al., 1988; Alawieh et al., 2018b).

\section{Intracranial hemorrhage (ICH) and mortality}

Survival studies were performed over $3 \mathrm{~d}$ or 30 -d duration after MicroE or MCAO stroke. Animals were euthanized if they demonstrated $>20 \%$ weight loss from baseline with inability to groom and reach for food, signs of severe distress, or signs of ICH (seizures, immobility, labored breathing, death). ICH was investigated by gross pathologic examination of coronal brain sections postmortem.

\section{Enriched environment (EE) exposure}

We used combined motor and cognitive rehabilitation in an EE as a model for poststroke rehabilitation. After $48 \mathrm{~h}$ of reperfusions, animals were randomly allocated to regular housing or EE. Animals in EE groups were housed in bigger multilevel cages with access to multiple toys including ladders, pipes, wooden toys of different shapes and sizes, and a running wheel. The items within the cage were changed twice weekly to ensure continuous exposure to novel stimuli. In addition to ladders and running wheels, $6-10$ pieces of $2.4-\mathrm{cm}$-long vermicelli pasta were added to the EE cages daily to stimulate forearm handling and movement. Animals not assigned to EE cages were housed in regular cages (30\% size of EE cages) with no access to toys, ladders or wheels, and were provided $0.4 \mathrm{~cm}$ long pieces of vermicelli that do not require forearm handling as described (Allred et al., 2008).

\section{Recovery measures}

Neurologic deficit scores were assessed using the modified neurologic severity scoring system in mice (mNSS) using a scoring system with increasing severity from 0 to 4 as described (Alawieh et al., 2018b) by two blinded observers. The corner task was used to assess forearm laterality and was performed at baseline and multiple time points after stroke (Zhang et al., 2002; Alawieh et al., 2018b). The ladder rung task was performed as previously described (Metz and Whishaw, 2009). In brief, animals were trained on using the ladder $7 \mathrm{~d}$ before stroke surgery. Following acclimation, animals were allowed to passively cross the ladder for three trials while being video recorded at $7 \mathrm{~d}$ before stroke, and on poststroke days 7,14 , and 21 . Videos were analyzed by blinded observers and each paw was given a score for placement between 1 and 6 as described (Metz and Whishaw, 2009): (1) for deep slip, (2) for slight slip, (3) for replacement, (4) for correction, (5) for partial placement, and (6) for correct placement. Average of all steps per limb was computed and a motor score for each limb, as well as a total score (sum of all four limb scores), was recorded. Barnes maze task was used to assess spatial learning and memory. For Barnes maze, animals were trained on identifying the location of the escape hole during the last week before euthanasia. Mice were given five training sessions, followed by one testing session $48 \mathrm{~h}$ later to assess retention as previously described (Alawieh et al., 2018b). Path length and number of error pokes were used to assess learning and memory. 


\section{Infarct size estimation}

For acute studies, infarct volume was calculated from TTC-stained 2$\mathrm{mm}$ brain slices using edema-corrected infarct volume, and result was reported as percentage infarct as described (Alawieh et al., 2018b). For chronic studies, Nissl stained serial brain sections $200 \mu \mathrm{m}$ apart and $40 \mu \mathrm{m}$ thick (see below for tissue processing) were used to reconstruct infarct, and the percentage infarct volume was computed as in acute studies (Alawieh et al., 2018b). Using NIH ImageJ, areas of neuronal cell loss on $40-\mu \mathrm{m}$-thick brain sections (five sections/mouse at stereological location: bregma $-0.58,-1.06,-1.58$, and -2.06$)$ were manually marked by two observers, along with the total area of ipsilateral and contralateral hemispheres. Infarct volume was then computed as the [area of contralateral hemisphere minus (area of ipsilateral hemisphere less the infarct area)] divided by the area of contralateral hemisphere. The average of both observers was reported for each animal.

\section{Tissue processing and histologic analyses}

For histologic analysis, immunohistochemistry, and immunofluorescence staining, brains were harvested at predetermined endpoints after intracardiac perfusion of experimental mice with ice-cold PBS followed by $4 \%$ paraformaldehyde (PFA) with infusion pump assistance. Brains were then cryopreserved in 30\% sucrose and then frozen and serially cut into $40-\mu \mathrm{m}$-thick sections. Sections that were $200 \mu \mathrm{m}$ apart were used for immunostaining. Both immunohistochemistry and immunofluorescence staining were performed as previously described (Alawieh et al., 2018b). Super-resolution imaging was performed using a Zeiss LSM 880 confocal microscope (Zeiss), and Airy Scan was used for high-resolution fields. Images were deconvoluted using ZEN 2.5 software (Zeiss), and then processed using Imaris 9.2 (Bitplane) for 3D reconstruction of imaged fields demonstrating relative positions of neurons, microglia and synapses. Surfaces were used to reconstruct neurons and microglia, and spots were used to localize individual synapses with spots localized to the center of each synaptic bud. Synaptic localization to microglia was analyzed using Imaris 9.2. The Ibal channel was used to create a mask representing the volume of individual microglia. Then, using a threshold of positive signal based on negative controls, spot analysis was used to detect individual synaptic buds from the 3D volume. This was followed by a filter that only included spots that fully overlapped with the microglial volume, and these spots were automatically represented and counted. All analyses were performed using automated modules and applied uniformly to all fields to avoid bias. For consistency across samples, a partial overlap between SV-2 and Iba1 signals is considered similar to full overlap when counting synaptic phagocytosis. In addition, we also computed a volumetric-based phagocytic index using automated analysis within Imaris. In brief, volume of microglia ( $\mathrm{Iba1}^{+}$stain) was reconstructed from a $40-\mu \mathrm{m}$ stack of high-resolution high-power fields in Imaris, and a channel was created to include only the overlap between Iba1 and SV-2 signals. This co-localization channel was also rendered as a volume. To compute the phagocytic index, the volume of synaptic material in the microglia was divided by the total volume of glia within the field, and reported as phagocytic index as a percentage. The primary antibodies used for staining were: anti-C3d (R\&D Systems, AF2655), anti-SV-2 (DHSB, AB_2315387), anti-Iba1 (Abcam, ab5076), anti-LAMP1 (Bio-Legends, 121609), anti-Mac2 (Abcam, AF1197), anti-Dcx (Abcam, ab18723), and anti-NeuN (Abcam, EPR12763).

\section{Immunostaining and imaging}

Extracted brains were cut into serial sections and stereological coordinates were used to identify concordant sections for staining from each group. The perilesional area was defined using stereotactic coordinates to ensure that uniform and comparable fields are chosen from each animal. To ensure unbiased stereology, slices from $\sim(-) 0.58$ and $(-) 1.58$ relative to bregma were imaged for each animal. Then the perilesional

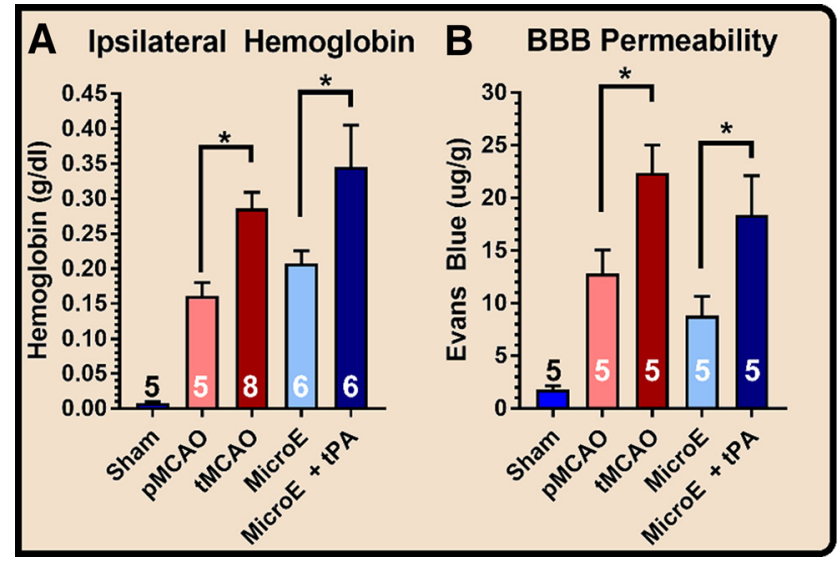

Figure 1. Reperfusion-induced $B B B$ dysfunction and hemorrhage after stroke. Ischemia was induced in adult (12-week-old) male C57BL/6 mice. $\boldsymbol{A}$, Hemoglobin content in the brain, comparing pMCAO to tMCAO in filamentous stroke model (mechanical reperfusion), and comparing t-PA treatment to vehicle in embolic stroke model (pharmacological reperfusion), at $24 \mathrm{~h}$ after ischemia. $\boldsymbol{B}, \mathrm{BBB}$ permeability measured by EB extravasation at $24 \mathrm{~h}$ after ischemia, comparing PMCAO to tMCAO in filamentous stroke model and t-PA treatment to vehicle in embolic stroke model. One-way ANOVA with Bonferroni's post hoc analysis comparing pMCA0 to tMCA0, and MicroE to MicroE + tPA; ${ }^{*} p<0.05$.

cortex, hippocampus, and basal ganglia were defined based on the expected edge of infarct in vehicle controls. The constraints of this area are reported on brain atlas schematics within the figure panels, and were uniformly used across all animals. Both immunohistochemistry and immunofluorescence staining was performed as described (Alawieh et al., 2018b). Super-resolution imaging was performed using a Zeiss LSM 880 confocal microscope with AiryScan (Zeiss). Images were deconvoluted using ZEN 2.5 software (Zeiss), and then processed using Imaris 9.2 (Bitplane) for 3D reconstruction.

\section{Human subjects}

Patient population

Patients admitted to the Medical University of South Carolina for mechanical thrombectomy (MT) or intravenous thrombolysis (using rt-PA) after AIS between January 2014 and December 2017 were, retrospectively, reviewed from a prospectively maintained registry of AIS outcomes. This study was approved by the Institutional Review Board (IRB) at each center.

\section{Thrombectomy or thrombolysis treatment}

Patients were treated with intravenous thrombolysis using rt-PA based on the most recent guidelines from the American Heart Association (Powers et al., 2019). Patients were selected for MT if after CT perfusion imaging they demonstrated a mismatch between cerebral blood volume and blood flow indicating a region of penumbra that is likely to explain their presenting National Institutes of Health Stroke Scale (NIHSS) score, as previously described (Alawieh et al., 2018d, 2019). Patients underwent MT regardless of age, type of occluded vessel, ASPECT score on CT imaging, or preprocedural administration of IV-tPA. MT was performed using the ADAPT (a direct aspiration at first pats Technique) as previously described (Turk et al., 2014). In brief, ADAPT was performed via direct aspiration of occlusive clot using the largest caliber aspiration device that the target vessel could accommodate. Devices used included the 5MAX, 5MAX ACE, ACE64, ACE68, 3MAX, or 4MAX (Penumbra). Aspiration was attempted three to four times before switching to alternative rescue therapy with a stent retriever at the discretion of the operating physician. Successful recanalization was assessed using the modified thrombolysis in cerebral ischemia (mTICI) scoring system by an experienced neuroradiologist. Achieving an mTICI score of $2 \mathrm{~B}$ or higher is considered as successful recanalization. 
A

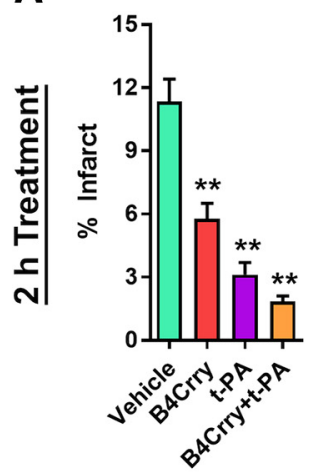

F
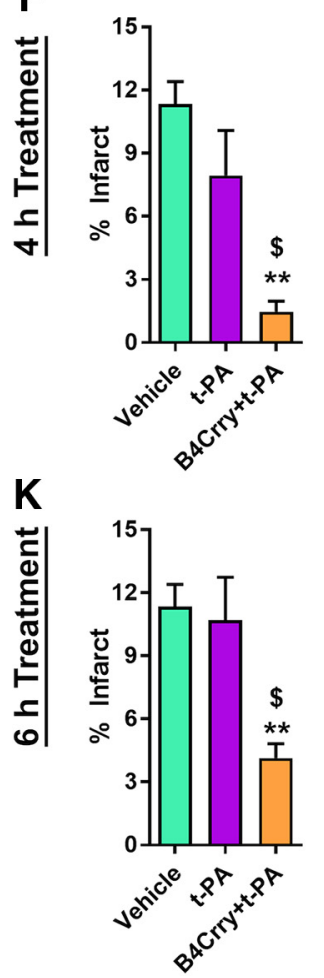

B

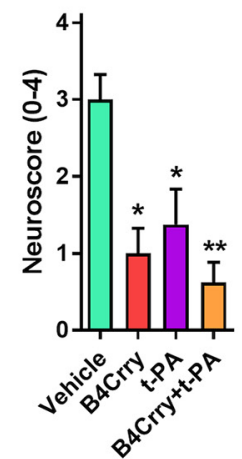

G

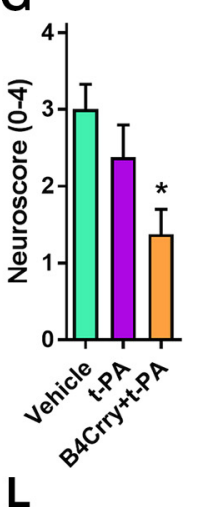

L

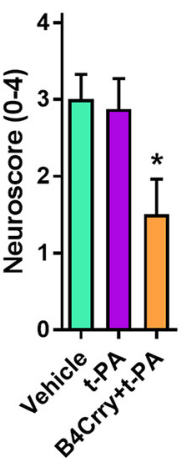

C

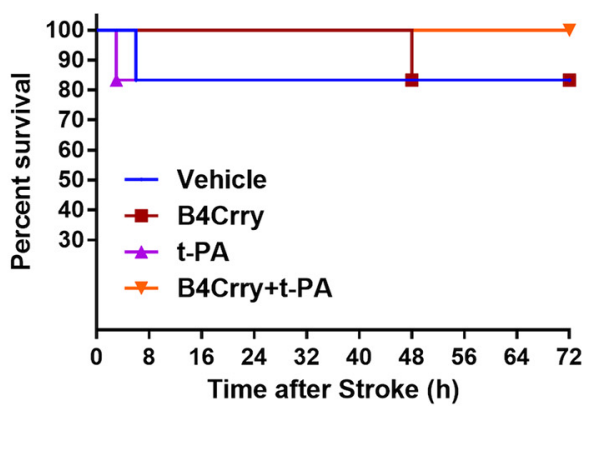

H

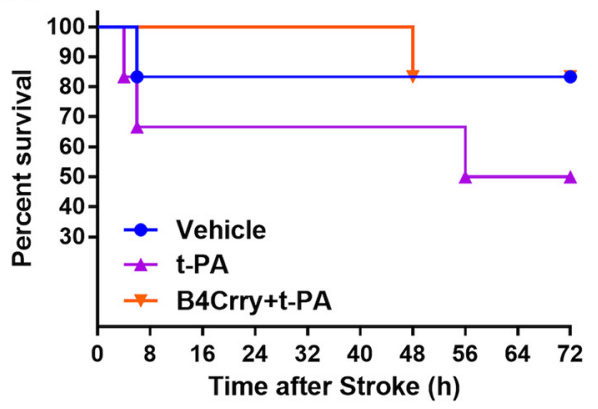

M

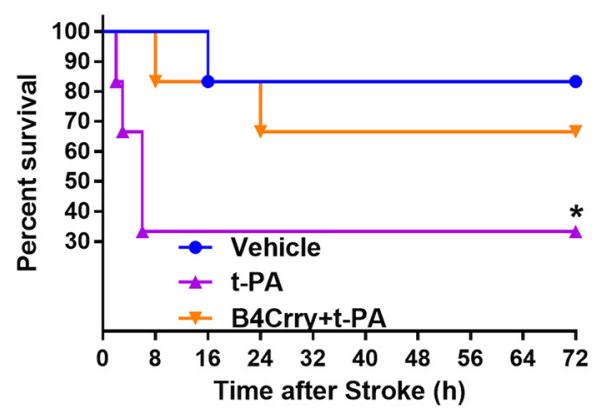

D

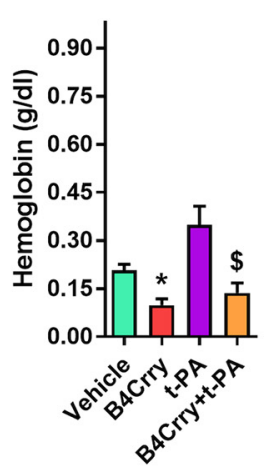

I
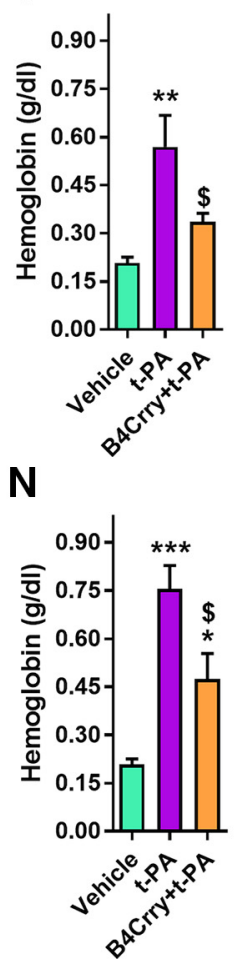

E

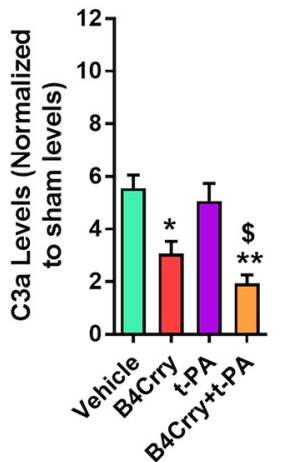

J

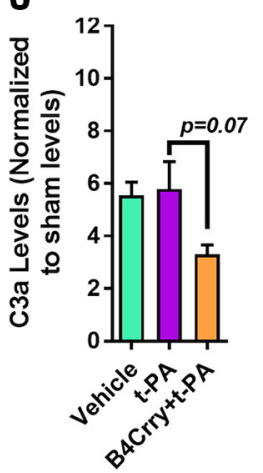

0

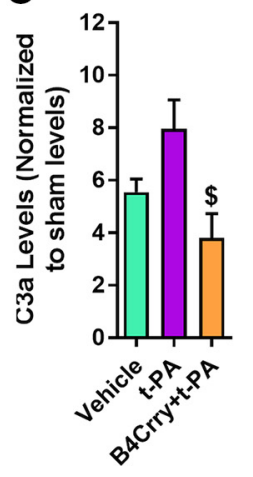

Figure 2. Complement inhibition improves safety and efficacy profile of t-PA after embolic stroke. Animals were subjected to high dose MicroE stroke and treated with vehicle, tPA, B4Crry, or a combination of B4Crry+t-PA at different time points after emboli injection $(\boldsymbol{A}-\boldsymbol{E}, \mathbf{2} \mathrm{h} ; \boldsymbol{F}-\boldsymbol{J}, 4 \mathrm{~h} ; \boldsymbol{K}-\mathbf{0}, \mathbf{6} \mathrm{h}$ ). $\boldsymbol{A}, \boldsymbol{B}$, Infarct volume (see also Extended Data Figure 2-1) and neurologic deficit scores at $24 \mathrm{~h}$ after MicroE stroke in animals treated at $2 \mathrm{~h}$ after ischemia. ANOVA with Bonferroni. $N=6 / g r o u p$. C, Kaplan-Meyer survival curve showing the survival among the four groups over $3 \mathrm{~d}$ of recovery. $\boldsymbol{D}, \boldsymbol{E}$, Hemoglobin content and C3a levels in ipsilateral brain hemisphere homogenates at $24 \mathrm{~h}$ after MicroE stroke. ANOVA with Bonferroni. $\boldsymbol{N}=6$ /group. $\boldsymbol{F}-\boldsymbol{J}$, Similar to $\boldsymbol{A}-\boldsymbol{E}$ but with animals treated at $4 \mathrm{~h}$ after MicroE stroke and assessed at same time points. $\boldsymbol{K}-\boldsymbol{0}$, Similar to $\boldsymbol{A}-\boldsymbol{E}$ but with animals treated at $6 \mathrm{~h}$ after MicroE stroke and assessed at same time points. Bars $=$ mean \pm SEM. Comparisons to vehicle: ${ }^{*} p<0.05,{ }^{* *} p<0.01,{ }^{* * *} p<0.001$. Comparisons to t-PA: $\$ p<0.05$. All pairwise combinations were tested on multiple comparison analyses for panels $\boldsymbol{A - 0}$. Significant findings are highlighted by ${ }^{*}$ or $\$$ signs. Comparisons to vehicle: ${ }^{*} p<0.05$, ${ }^{* *} p<0.01,{ }^{* *} p<0.001$. Comparisons to t-PA: $\$ p<0.05$. Extended Data Figure $2-2$ shows the effect of complement inhibition on intracerebral hemorrhage after mechanical reperfusion using tMCAO model.

\section{Inclusion/exclusion criteria}

In addition to eligibility for intervention as determined above, this current study excluded patients who were admitted after intubation, which precludes a pretreatment assessment of cognitive and motor baseline, and only patients with full NIHSS assessment performed at baseline demonstrating both cognitive and sensorimotor impairment were included (at least two points on Cog-4 scale and two points on motor deficits). Patients with cognitive or motor deficits at baseline (prestroke) were excluded from analyses.

\section{Data collection}

Patient charts were reviewed for patient demographics, presence of one of the major stroke comorbidities including diabetes, hypertension, atrial fibrillation or hyperlipidemia, the modified Rankin score (mRS) at baseline, NIHSS at admission, and the treatment received including whether IV-tPA was administered. MT procedure notes were reviewed for procedural variables including the final TICI score and onset-toreperfusion time.

\section{Clinical outcomes}

Clinical outcomes were assessed on direct evaluation of patients by a stroke neurologist. Cognitive assessment at presentation was determined by the Cog-4 component of the NIHSS as previously described. and included the components (1b, 1c, 9, and 11) with a score range of 0-9 (Hajjar et al., 2013). Motor assessment was computed from the sensorimotor components of the NIHSS, including components $4,5,6$, and 8 (facial paralysis, limb paralysis, dysarthria). Postdischarge follow-up outcomes were collected at $90 \mathrm{~d}$ after stroke 


\section{Standard Care}
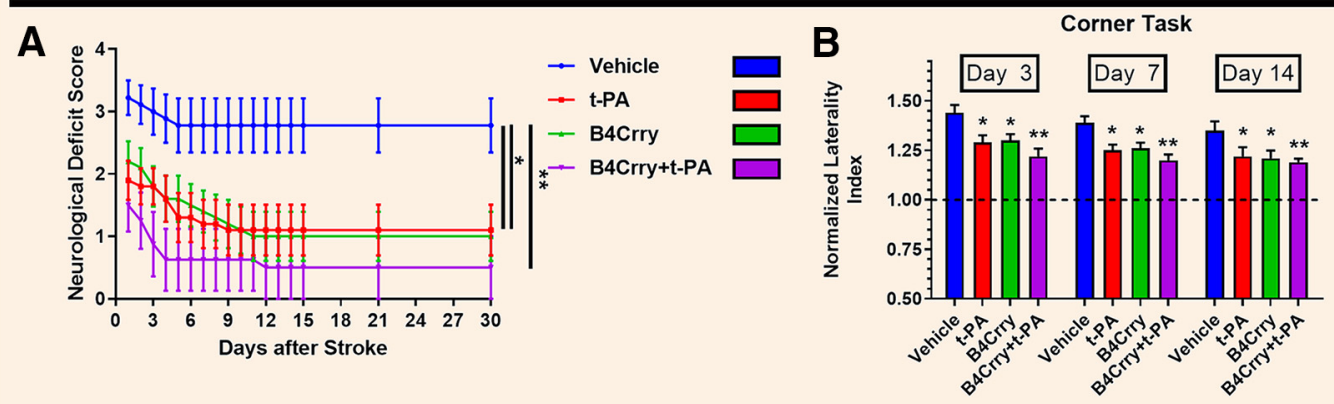

C

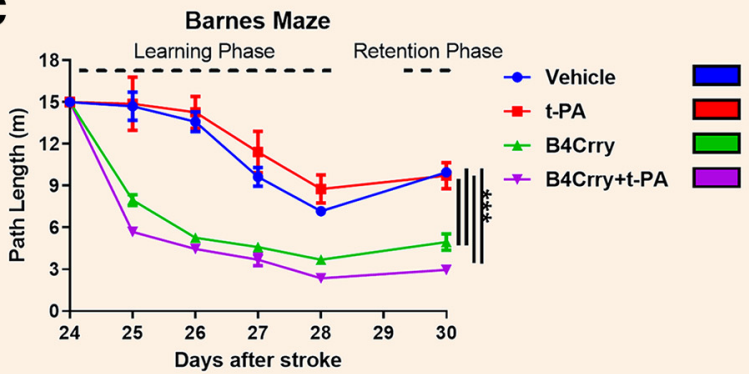

D Barnes Maze

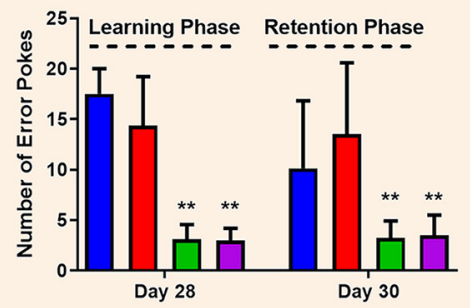

Days after stroke

\section{Rehabilitation}
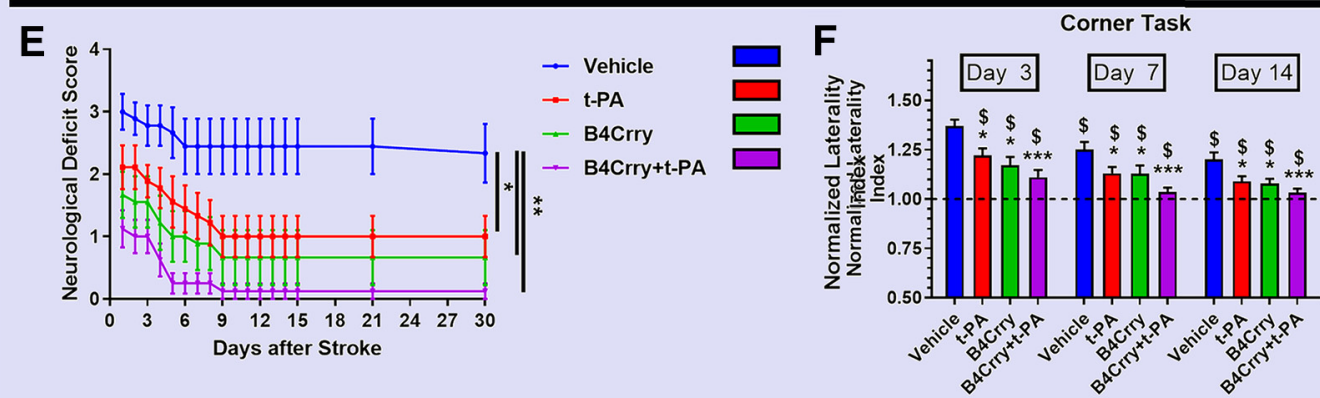

G

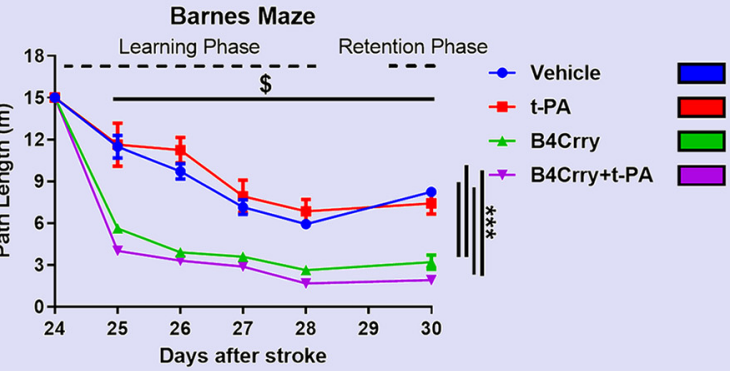

H

Barnes Maze

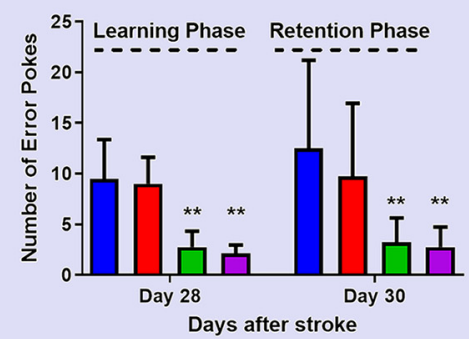

I

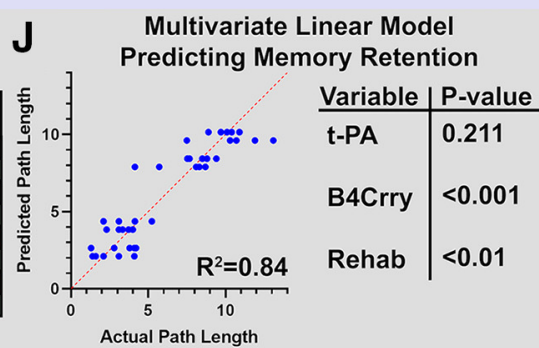

Figure 3. Cognitive and motor recovery over a 30-d period after stroke, with acute t-PA, B4Crry, or combined treatment in the context of either spontaneous recovery or rehabilitation therapy. Animals subjected to MicroE were treated with t-PA, B4Crry, or combined B4Crry +t-PA at $2 \mathrm{~h}$ after emboli injection, and animal behavioral tests performed over $30 \mathrm{~d}$. $\boldsymbol{A}-\boldsymbol{D}$, Animals were housed in standard cages with minimal enrichment (no rehabilitation; see Materials and Methods) for duration of experiment. Neurologic deficit scores $(\boldsymbol{A})$ and measurement $(\boldsymbol{B})$ of normalized laterality index on corner test after MicroE and $30 \mathrm{~d}$ of recovery. Two-way ANOVA with Bonferroni. $N=9-10 /$ group; ${ }^{*} p<0.05$ and ${ }^{* *} p<0.01$, comparisons to vehicle. Extended Data Figure 3-2 shows individual neurologic deficit data without using the last-observation carried forward paradigm, and the Hedges' $g$ index for effect size. Performance on ladder rung task showed a similar pattern of recovery across groups and is shown in Extended Data Figure 3-3. C, D, Barnes maze test: animals treated with vehicle or B4Crry were trained on finding the escape hole on 
$( \pm 14)$ during follow-up visits with a stroke neurologist or nurse practitioner. Each subject served as his/her own control, and cognitive recovery for each subject was compared with recovery of sensorimotor deficits based on percentage improvement from baseline. We also compared motor and cognitive recovery among patients who underwent thrombectomy with versus unsuccessful recanalization. Successful recanalization was defined as a score of $2 \mathrm{~B}$ or higher on mTICI scale (mTICI score).

\section{Statistical analysis}

Statistical analyses were performed using GraphPad Prism 6.0 (GraphPad) and SPSS v24 (IBM Corporation). Parametric testing was used unless otherwise specified in the event of significant BrownForsythe test for homogeneity of variance or if normality fails. Nonparametric testing was alternatively used as indicated in figure legends. Neurologic deficit scores were compared using non-parametric $t$ tests. Group analyses were performed using one-way ANOVA (or two-way ANOVA for multiple time points) followed by multiple comparison analyses (Bonferroni), or using repeated measures ANOVA for matched data; $p$ values below 0.05 were considered significant. Student's $t$ test (parametric) or Mann-Whitney tests (nonparametric) was used to compare two groups and was always used as two-tailed. Multivariate regression analyses were performed using SPSS v24 (IBM Corporation).

\section{Results}

\section{Mechanical or pharmacological reperfusion therapy} exacerbates poststroke hemorrhage and BBB leakage

To assess how cerebral reperfusion affects ICH and BBB dysfunction after stroke, we used two murine models of IS; the tMCAO model and a MicroE. Both mechanical withdrawal of intraluminal occlusive filament (tMCAO) or administration of t-PA after MicroE increased hemoglobin content and EB leakage across the $\mathrm{BBB}$ at $24 \mathrm{~h}$ after stroke (Fig. 1A,B). To compare the two reperfusion strategies, we additionally used a pMCAO model, and demonstrated that the effect size of mechanical reperfusion in tMCAO compared pMCAO was greater than that between pharmacological reperfusion and vehicle treatment in MicroE stroke (Hedges' $g$ index: 2 vs 1.2, respectively, for hemoglobin and 1.6 vs 1.3 for EB extravasation; Fig. $1 A, B)$.

days 24-28 after MicroE. The path length to find the escape hole during training was computed using automated video analysis. The number of error pokes (nose pokes into non-target holes) was also computed. Retention of learned memory was assessed on day 30. Repeated measure two-way ANOVA with Bonferroni. $N=8 /$ group; ${ }^{* *} p<0.001$, comparison to vehicle and t-PA. $\boldsymbol{E}-\boldsymbol{H}$, Animals were assigned to rehabilitation cages on day 2 after MicroE (see methods), and the same analyses were performed as in $\boldsymbol{A}-\boldsymbol{D}$; ${ }^{*} p<0.05$, ${ }^{* *} p<0.01$, ${ }^{* *} p<0.001$ comparisons within the four groups subjected to rehab. $\$ p<0.05$ compared with the corresponding groups in standard care category $(\boldsymbol{A}-\boldsymbol{D})$ ). Bars $=$ mean \pm SEM. Note: within each care group (standard or rehab), all pairwise combinations were tested on multiple comparison analyses to compare treatment groups (vehicle, $t-P A$, $\mathrm{B} 4$ (rry, B4(rry+t-PA). Only findings that were significant are highlighted with *. Comparing care groups (standard vs rehab); $\$$ denotes $p<0.05$ when comparing corresponding treatment groups within each care category (e.g., vehicle vs vehicle + rehab, tPA vs tPA+ rehab). Bars $=$ mean \pm SEM. $I$, Summary table of the impact of each intervention on the battery of motor and cognitive tasks performed. $(-)$ denotes no effect and $(+)$ denotes a significant improvement compared with vehicle. Red signs denote significant effect in Rehab compared to standard care. $\boldsymbol{J}$, Multivariate linear regression model for prediction retention memory (path length) showing that B4Crry and rehab are independent predictors, with no effect for t-PA. B4Crry had significantly higher effect (Wald's $=-5.8, p<0.001$ ) compared with rehabilitation (Wald's $=-1.72, p<0.01$ ) in predicting path length. Pearson's correlation for the model, $R^{2}=0.84$. See Extended Data Figure 3-1 for survival data. B4Crry did not have any impact on cognitive recovery in sham animals, as shown in Extended Data Figure 3-4.

\section{Localized complement inhibition limits t-PA associated hemorrhage and increases the safety profile and treatment window for thrombolytic therapy}

To investigate the interaction between complement modulation and intravenous thrombolysis, we focused on the MircoE stroke model, a less studied model than $\mathrm{MMCAO}$, but more relevant for investigations involving intravenous thrombolytic therapy. $\mathrm{t}-\mathrm{PA}$ administered $2 \mathrm{~h}$ after microemboli injection reduced infarct volume and neurologic deficit compared with vehicle-treated controls, as did 2-h administration of B4Crry or a combination of B4Crry+t-PA (Fig. 2A,B; Extended Data Fig. 2-1). There was no difference in acute mortality between the groups (Fig. 2C). B4Crry significantly reduced hemoglobin content $24 \mathrm{~h}$ after stroke compared with vehicle (Fig. $2 D$ ), whereas t-PA treatment was associated with significantly higher hemoglobin content compared with B4Crry treatment. Administration of B4Crry together with $\mathrm{t}-\mathrm{PA}$ reversed the $\mathrm{t}$-PA-mediated increase in brain hemoglobin content (Fig. 2D). To confirm that B4Crry treatment inhibited complement activation in the ischemic hemisphere, we demonstrated that B4Crry and B4Crry+t-PA, but not $\mathrm{t}-\mathrm{PA}$ alone, reduced $\mathrm{C} 3 \mathrm{a}$ levels in the ipsilateral hemisphere compared with vehicle (Fig. 2E).

We next investigated how delayed administration of t-PA, with and without B4Crry, affects poststroke outcomes. When t-PA administration was delayed, we observed a significant increase in mortality in the 6-h group compared with vehicle. This increase in mortality was reversed when t-PA was coadministered with B4Crry (Fig. 2F-O). The increased mortality in t-PA-treated animals was associated with a significant increase in brain hemoglobin content compared with vehicle (Fig. 2I,N), and t-PA alone had no significant inhibitory effect on C3a generation in the ipsilateral hemisphere (Fig. 2J,O). On the other hand, co-administration of B4Crry with t-PA significantly reduced t-PA-associated cerebral hemorrhage at both 4 and $6 \mathrm{~h}$ of administration time points, and significantly reduced the level of C3a generated at the 6-h time point (Fig. 2IJ,N,O). At 4- and 6-h treatment schedules, only combination treatment (B4Crry+ t-PA) reduced neurologic deficits and infarct volume after MicroE (Fig. 2F,G,K,L; Extended Data Fig. 2-1). These findings indicate that t-PA is associated with increased reperfusioninduced hemoglobin extravasation after stroke, an effect that becomes more pronounced as the time of administration of t-PA is increased beyond $2 \mathrm{~h}$ of onset. Complement activation after tPA administration adversely impacts the safety and efficacy profile of thrombolytic therapy, as indicated by the protective effect of B4Crry. Notably, we also observed a B4Crry dose-dependent reduction of hemoglobin content in the brain, that corresponded to rates of ICH-induced death occurring after mechanical reperfusion (i.e., removal of filament after 1-h ischemia in tMCAO model) and B4Crry administration (Extended Data Fig. 2-2).

\section{Thrombolytic therapy improves motor, but not cognitive recovery, a dissociative effect eliminated with complement inhibition}

We next assessed long-term motor and cognitive recovery in mice treated with t-PA, B4Crry or B4Crry+t-PA at $2 \mathrm{~h}$ after emboli injection. There was no difference in survival between animals treated with $\mathrm{t}$-PA versus animals treated with vehicle over $30 \mathrm{~d}$ (Fig. 3; Extended Data Fig. 3-1), but animals treated with t-PA showed early significant improvement in neurologic deficits, forearm laterality (corner task), and ladder rung task (Fig. 3; Extended Data Fig. 3-3). Motor deficits continued to improve in t-PA-treated mice over the first two weeks, whereas 

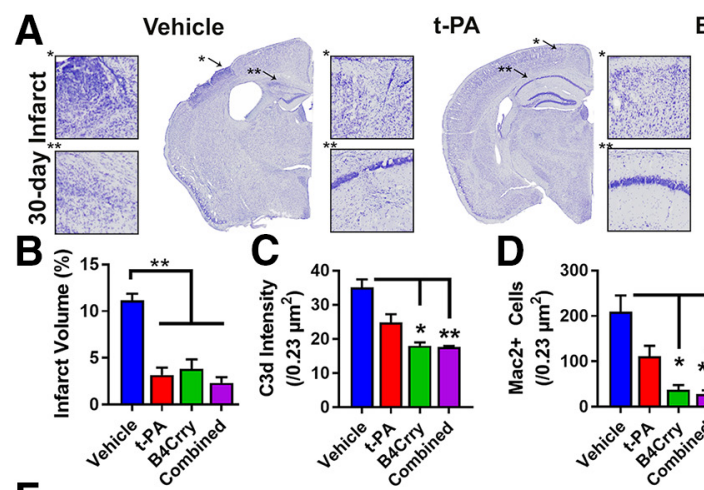

C

D
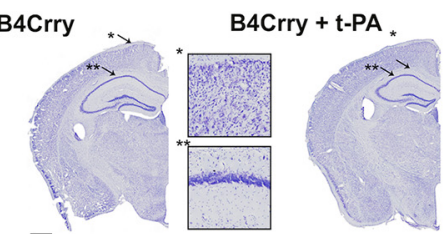

$\mathbf{F}$
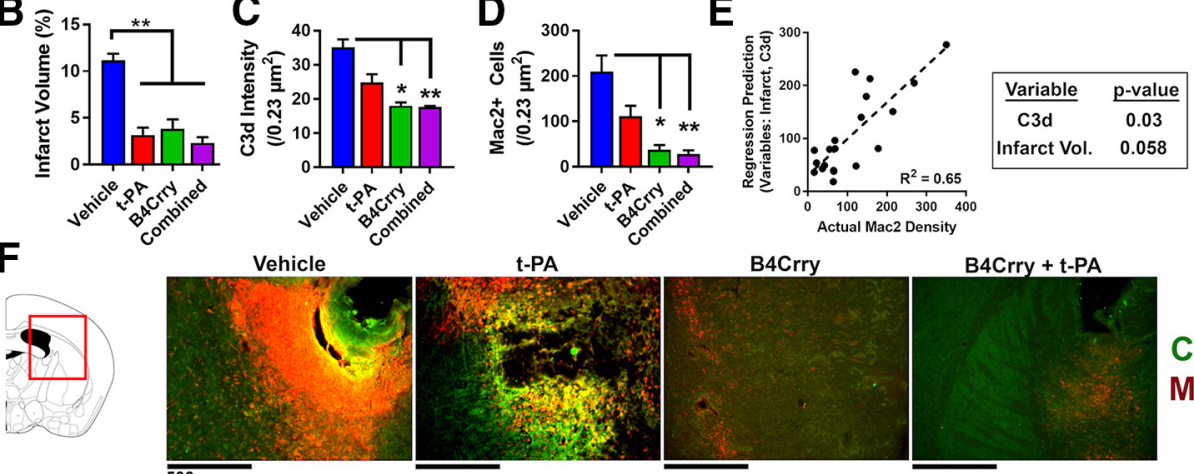

C3d I

MAC2
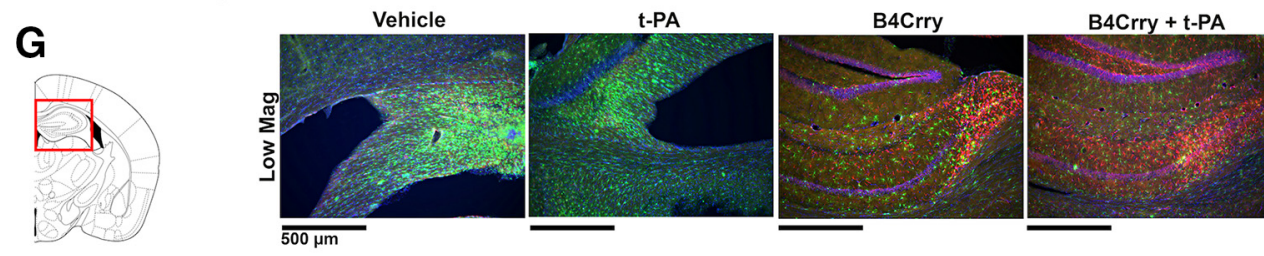

Iba1 |

Dcx I

DAPI
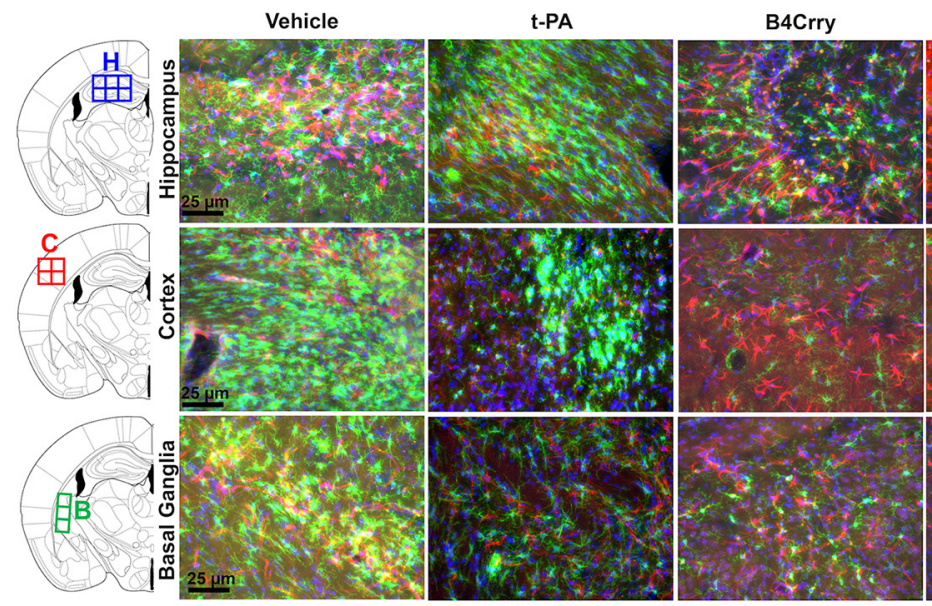

B4Crry + t-PA

Sham
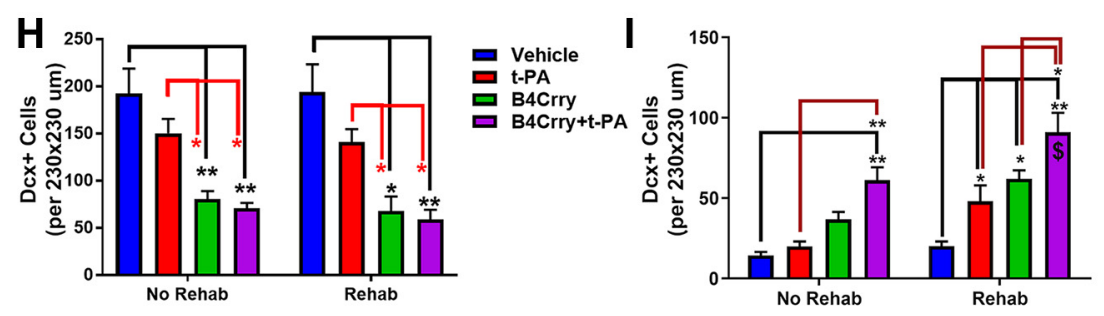

Figure 4. Impact of infarct size and complement activation on microgliosis and migration of neuronal progenitor cells after stroke. Mice subjected to MicroE stroke were treated with vehicle, $\mathrm{t}-\mathrm{PA}$, or B4Crry + t-PA at $2 \mathrm{~h}$ after emboli injection, and brains isolated $30 \mathrm{~d}$ later. A, Niss-stained sections of brains from each group showing regions of neuronal loss. B, Quantification of lesion volume across four groups. $C, D$, Quantification of mean intensity of C3d deposition and $\mathrm{Mac2}{ }^{+}$cells in the perilesional brain. One-way ANOVA with Bonferroni. $N=5 / \mathrm{group} ;{ }^{*} p<0.05$, ${ }^{* *} p<0.01$. $E$, Multivariate linear regression model predicting the density of $\mathrm{MaC}^{+}$cells using $33 \mathrm{~d}$ deposition and infarct volume as co-variates. Pearson's correlation $R^{2}=0.65, p<0.0001$. $N=20$. $\boldsymbol{F}$, Representative fields of perilesional brains quantified in C, D showing C3d (green) and MAC2 (red) staining. G, IF staining of Dcx (red) and Iba1 ${ }^{+}$(green) cells, with DAPI staining (blue), in the perilesional brain at $30 \mathrm{~d}$ after MicroE. Left panels show the stereotactic locations from which random fields were chosen. $\boldsymbol{H}, \boldsymbol{I}$, Quantification of $\boldsymbol{G}$. One-way ANOVA with Bonferroni. $N=5$ /group; ${ }^{*} p<0.05,{ }^{* *} p<0.01$. Bars = mean \pm SEM. All pair wise comparisons within rehab and no rehab groups were performed, only results that are significant are highlighted with *. Comparisons were also made within each treatment group comparing rehab to no rehab, and significant findings are highlighted by the $\$$ sign. 
A
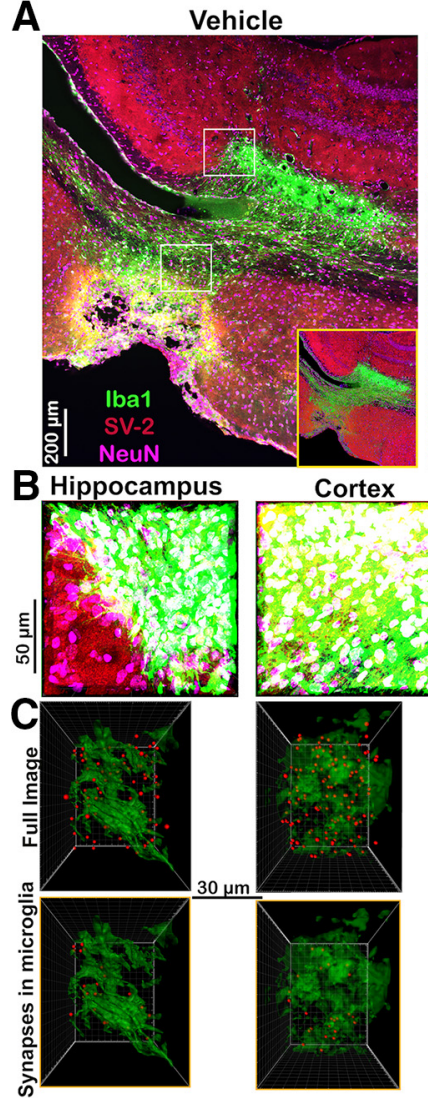

D
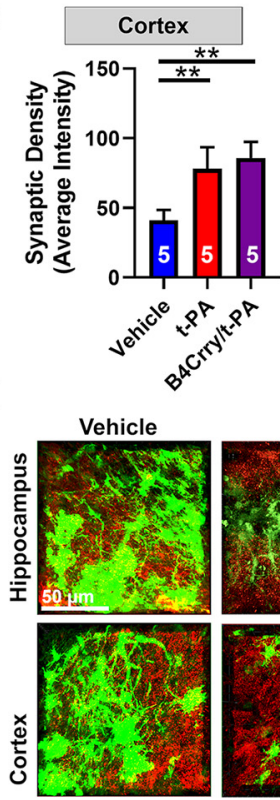

Cortex
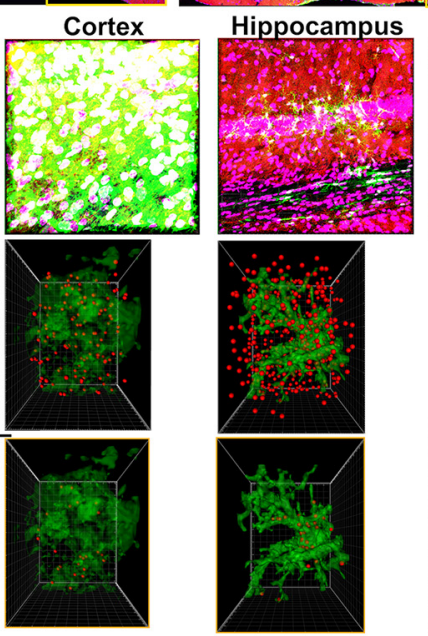

E

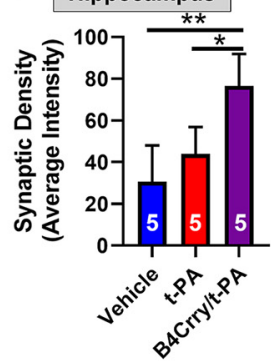

t-PA
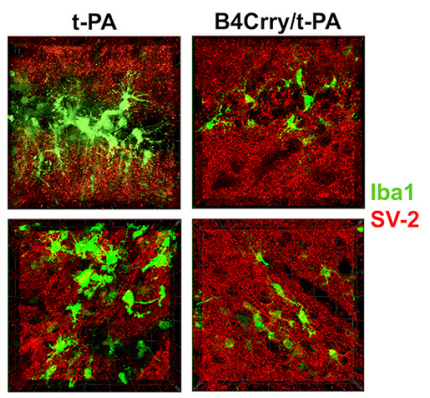

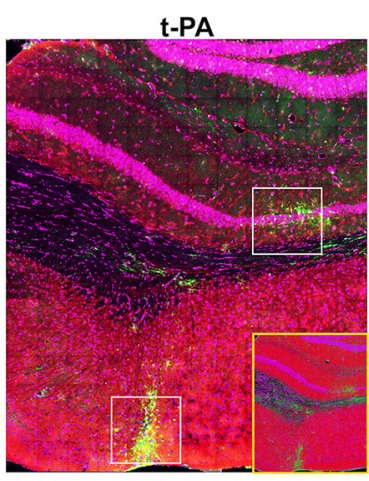

Cortex
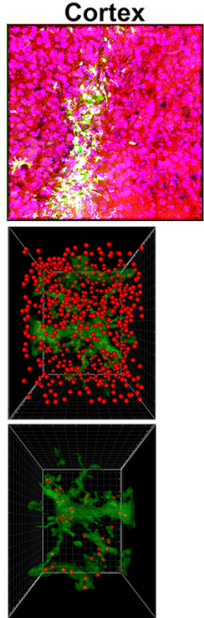

F

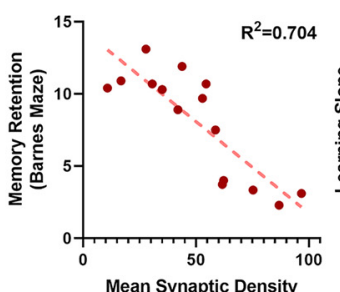

G

condary Contro

NeuN

anti-goat IgG-FITC
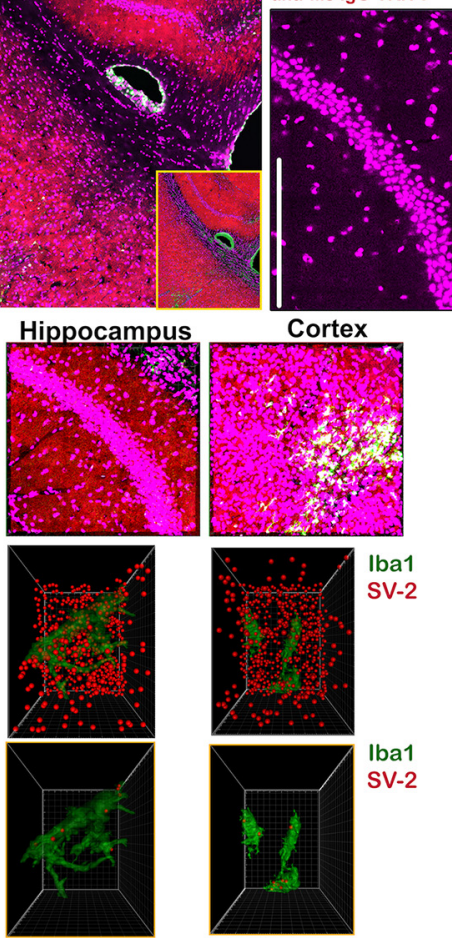

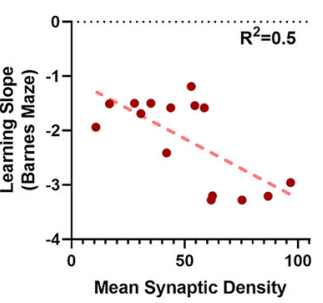

I
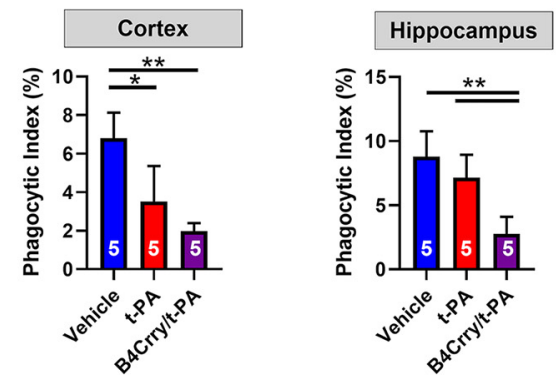

Figure 5. Complement inhibition but not reperfusion therapy prevents degeneration of perilesional hippocampal synapses after stroke. Mice subjected to MicroE stroke were treated with vehicle, t-PA, or B4Crry +t-PA at $2 \mathrm{~h}$ after emboli injection, and brains isolated $30 \mathrm{~d}$ later. $\boldsymbol{A}$, Large representative fields of the perilesional brain from the three treatment groups, depicting changes in microgliosis and synaptic density patterns between the groups in both the ipsilateral cortex and hippocampus (Iba1, green; SV-2, red; NeuN, pink). Right panel, Secondary antibody only control, representing slices stained with NeuN, anti-mouse IgG-TRITC (secondary control for SV-2) and anti-goat IgG-FITC (secondary control for Iba1). B, High-resolution IF fields from cortex and hippocampus in $\boldsymbol{A}$. C, 3D reconstruction of microglia within the fields of $\boldsymbol{B}$ showing changes in synaptic density (SV-2, red; top panels) and the density of synaptic material in microglia (lba1, green; lower panels) across the different locations and treatment groups. D, $\boldsymbol{E}$, Quantification of high-resolution fields showing significantly higher density of synapses in the cortex of t$P A$ and B4Crry+t-PA-treated animals compared with vehicle treated $(\boldsymbol{D})$, with no difference between the t-PA and B4Crry $+\mathrm{t}-\mathrm{PA}$ groups. Synaptic density in the hippocampus $(\boldsymbol{E})$, however, was significantly higher in B4Crry + t-PA-treated mice compare to both t-PA and vehicle. ANOVA with Bonferroni; ${ }^{*} p<0.05,{ }^{* *} p<0.01$. $\boldsymbol{F}, \mathbf{G}$, Correlation of synaptic density with performance of animals on Barnes maze task. $\boldsymbol{F}$, Correlation of synaptic density with path length. $\mathbf{G}$, Correlation of synaptic density with the slope of change in path length during training, a surrogate measure for the speed of learning or acquisition of the task. Pearson correlation coefficients were obtained, $R^{2}=0.7(\boldsymbol{F})$ and $0.5(\boldsymbol{G})$ and $p<0.0001$ for correlation. Extended Data Figure $5-1$ shows the change of microglia and synaptic density spatially in relation to the edge of the gliotic scar across the different treatment groups. $\boldsymbol{H}$, High-resolution stack of 40 - $\mu$ m slices showing the overlap between SV-2 and Iba1 staining. Representative sections from each group. Scale bar $=50 \mu \mathrm{m}$. I, Quantification of volume-based phagocytic index between the different groups 
vehicle controls showed minimal improvement in the motor domain (Fig. 3A,B; Extended Data Fig. 3-3). On the other hand, tPA alone did not improve outcome on Barnes maze cognitive task over $30 \mathrm{~d}$ of recovery, and t-PA-treated animals were indistinguishable from vehicle-treated controls during learning and retention phases (Fig. $3 C, D$ ). To rule out any independent effect of B4Crry, we demonstrated that there was no difference in cognitive outcomes between vehicle and B4Crry-treated mice that underwent sham surgery (Extended Data Fig. 3-4).

In the same paradigm, we investigated the effect of t-PA on motor and cognitive outcomes in the context of B4Crry therapy. Similar to t-PA alone, treatment with B4Crry or B4Crry $+\mathrm{t}-\mathrm{PA}$ reduced functional deficits over $30 \mathrm{~d}$ of recovery (Fig. $3 A, B$; Extended Data Fig. 3-3). We also compared the effect size of different interventions to vehicle using the Hedges' $g$ index to show that the effect of B4Crry+t-PA on motor recovery was higher than either intervention alone (Extended Data Fig. 3-2).

In contrast to t-PA alone, B4Crry and B4Crry+t-PA treatment significantly and similarly improved spatial learning and retention of learned memory during the last week of follow-up as compared with vehicle treatment (Fig. $3 C, D$ ). These findings indicate that complement inhibition with B4Crry has a synergistic effect with t-PA on motor recovery, and eliminates the motor-cognitive dissociation in the pattern of recovery, thus optimizing the response to t-PA.

\section{Rehabilitation does not compensate for complement-driven cognitive decline in t-PA-treated mice}

We next assessed whether t-PA therapy combined with rehabilitation, a standard of care, affects motor recovery, and whether rehabilitation can alleviate the deficit seen in cognitive recovery with t-PA treatment. With all treatments, survival and functional recovery was similar whether rehabilitation was included in the paradigm or not (Fig. 3 compare $A, B$ with $E, F$ ). Rehabilitation was associated with a more significant and faster recovery of forearm laterality on corner task in all treatment groups as compared with spontaneous recovery (no rehabilitation; Fig. 3 compare $B, F)$. On Barnes maze cognitive task, rehabilitation was also associated with improved spatial learning and retention of learned memory as compared with spontaneous recovery. However, B4Crry and B4Crry+t-PA groups still showed a significantly better learning and retention pattern on Barnes maze compared with t-PA or vehicle (Fig. $3 G, H$ ). With or without rehabilitation, there were no significant differences in performance on Barnes maze between vehicle and t-PA alone. These findings indicate that complement inhibition significantly improves cognitive recovery in mice treated with $\mathrm{t}-\mathrm{PA}$, beyond the trajectory of spontaneous and rehabilitation-induced recovery. Figure $3 I$ shows a summative analysis of these findings. Only B4Crry treatment and rehabilitation were independent predictors of shorter path length on Barnes maze retention, with a higher impact of B4Crry compared with rehabilitation (Wald's coefficients of -5.8 vs -1.72 ; Fig. $3 J$ ). We also investigated the performance of mice on learning and retention of avoidance memory using the passive avoidance task (Extended Data Fig. 3-3). Animals treated with B4Crry or combination therapy, but not t-

\footnotetext{
$\leftarrow$

showing significant reduction in phagocytic index in the hippocampus with combined treatment compared with t-PA or vehicle treatment. ANOVA with Bonferroni; ${ }^{*} p<0.05$, ${ }^{* *} p<0.01$. For all panels, all pairwise comparisons were performed, only significant results are highlighted with *.
}

PA. showed significantly better avoidance learning compared with vehicle at 15 and $21 \mathrm{~d}$ after MCAO (Extended Data Fig. 3-3).

\section{Chronic inflammatory microgliosis is promoted by complement activation}

We previously showed that acute complement inhibition interrupts an otherwise ongoing inflammatory cascade and potentiates reparatory mechanisms (Alawieh et al., 2015b, 2018b,c). Therefore, since reperfusion therapy reduces acute neuronal loss after stroke but does not improve cognitive outcome, we investigated the hypothesis that reperfusion therapy does not interrupt neuorinflammation in the reperfused brain. We first demonstrated that compared with the control group, t-PA, B4Crry, and $\mathrm{B} 4 \mathrm{Crry}+\mathrm{t}-\mathrm{PA}$ treatment groups all had a comparable reduction in infarct volume $30 \mathrm{~d}$ after MicroE stroke (Fig. 4A,B). However, animals treated with vehicle or t-PA alone had a significantly higher levels of complement $\mathrm{C} 3 \mathrm{~d}$ deposition in the ipsilateral brain compared with B4Crry groups, and C3d deposition was associated with migration of proliferating $\left(\mathrm{Mac}^{+}\right)$microglia/ macrophages and a higher density of activated microglia ( $\mathrm{Iba}^{+}{ }^{+}$; Fig. 3C-F). On multivariate analysis, C3d deposition was an independent predictor of the density of $\mathrm{Mac}^{+}$microglia/macrophages when adjusted for infarct volume (Fig. 4E). Microgliosis was found to track sites of complement C3d deposition in the perilesional brain (Fig. 4F).

\section{Relationship between complement-driven microgliosis, neurogenesis, and cognitive recovery}

Since neurogenesis is associated with improved performance in cognitive tasks, we assessed whether the lack of cognitive recovery in animals treated with t-PA is related to decreased neurogenesis and neuronal migration to the perilesional brain. t-PA treatment was associated with a higher density of $\mathrm{Ibal}^{+}$cells compared with B4Crry and B4Crry+t-PA treatments (Fig. 4G, $H)$. However, the ipsilesional brain from t-PA-treated mice did not have a lower density of neuroblast cells $\left(\mathrm{Dcx}^{+}\right)$compared with B4Crry treatment, despite better cognitive performance with B4Crry treatment (Fig. 4I). Furthermore, although B4Crry alone enhanced cognitive recovery compared with vehicle, it only enhanced poststroke neurogenesis and neuroblast migration when combined with either t-PA (reperfusion) or rehabilitation (Fig. 4I). The absence of differences between B4Crry alone and t-PA alone treatments in $\mathrm{Dcx}^{+}$cell density demonstrates that the difference seen in cognitive recovery between the two groups is not explained by neurogenesis.

\section{Complement mediated microglial phagocytosis of synapses occurs poststroke and is unaffected by reperfusion therapy} Data from models of neurodegenerative disease have implicated microglial-phagocytosis of synaptic connections and reduced synaptic density in cognitive dysfunction (Stephan et al., 2012; Vasek et al., 2016; Presumey et al., 2017). We therefore evaluated the interactions between chronic microgliosis, complement activation and synaptic density across different treatment groups. We focused on comparing t-PA and B4Crry+t-PA treatment groups, since these groups had comparable infarct volumes, but the largest differences in the pattern of recovery. We performed high-resolution immunofluorescent staining for microglia/macrophages (Iba1), synapses (SV-2), and neurons (NeuN) on perilesional brain sections at $30 \mathrm{~d}$ after MicroE (Fig. 5A). Brain sections were obtained from comparable stereological coordinates and assessed for synaptic density in both perilesional hippocampus and cortex. Both t-PA and B4Crry+t-PA-treated 
A

Iba1 | SV2 | NeuN

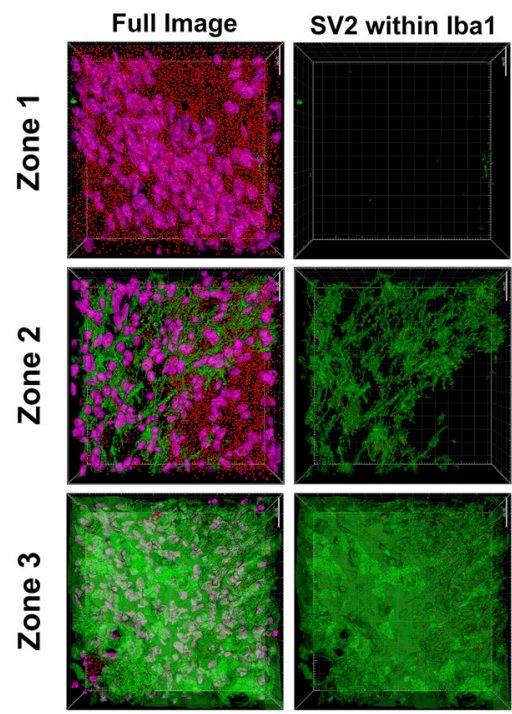

F

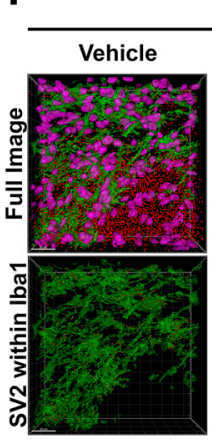

Zone 2

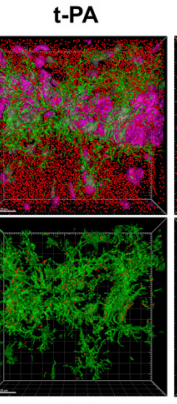

Iba1 | SV2 | NeuN

$\mathbf{G}$

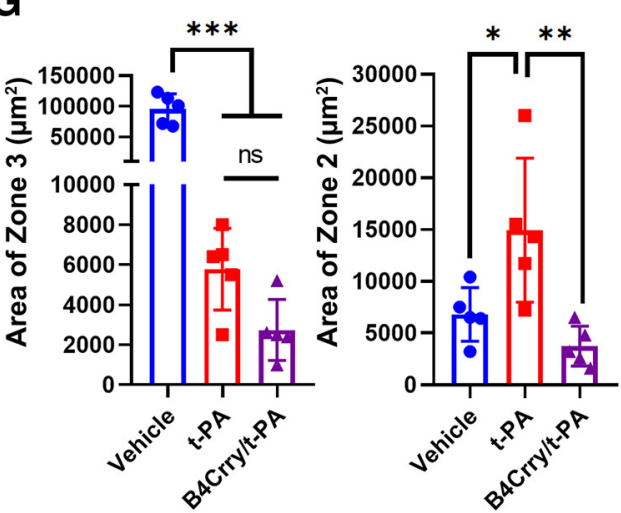

B

C
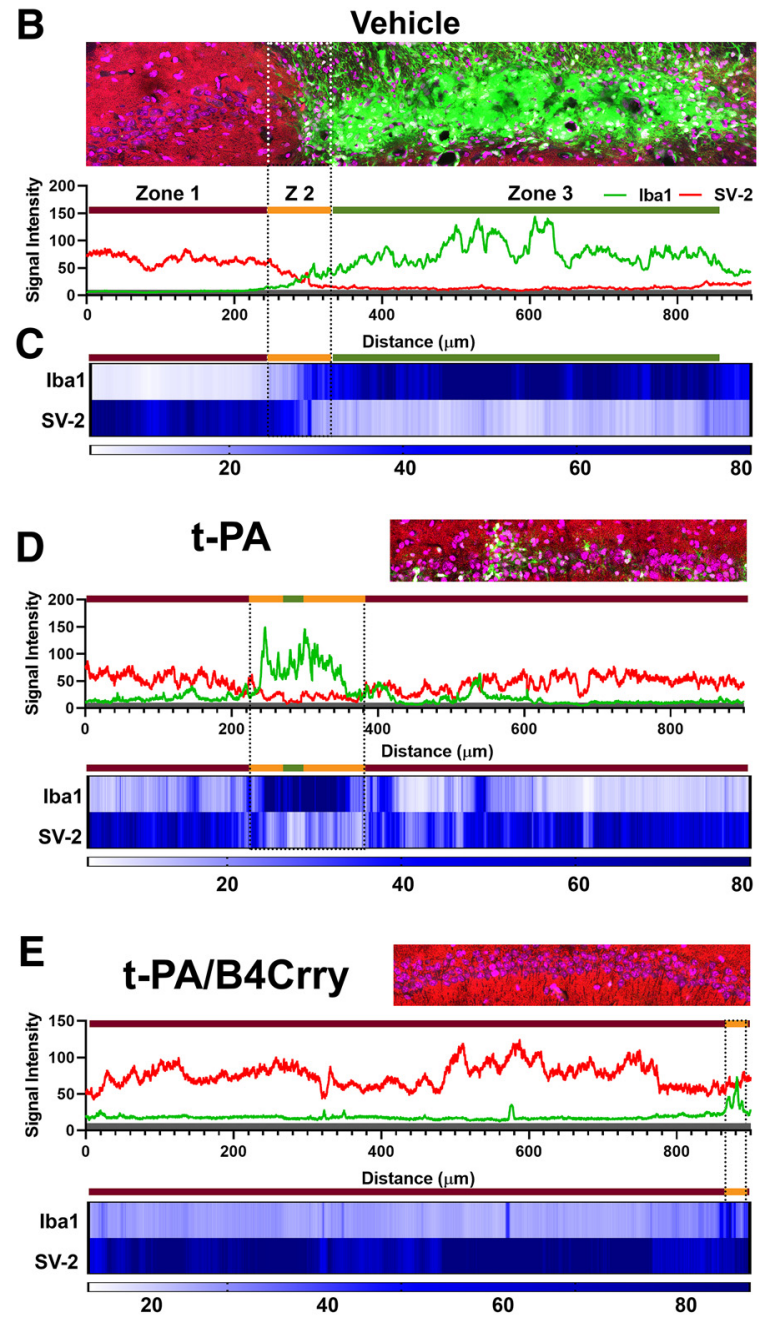

Zone 2

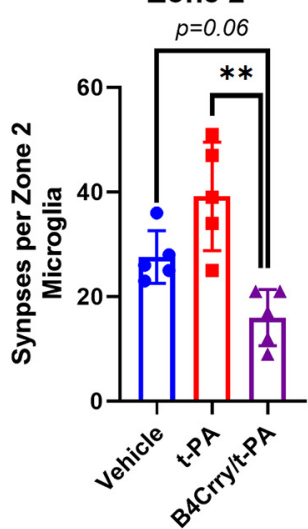

Figure 6. Complement inhibition reduces the extent of microglia engulfment of hippocampal synapses. Mice subjected to MicroE stroke were treated with vehicle, $t-P A$, or B4Crry $+t-P A$ at $2 \mathrm{~h}$ after emboli injection, and brains isolated $15 \mathrm{~d}$ later. $\boldsymbol{A}$, Perilesional microgliosis is classified into three zones: zone 1 denoting areas of baseline synaptic density and only occasional presence of Iba1+ cells, zone 2 denoting areas of 20-60\% loss of synaptic density with increasing microglia activity and surge in density of SV-2 particles in microglia (Fig. 5D), and zone 3, denoting loss of synaptic density and gliotic scar formation. Right, Examples of $3 \mathrm{D}$ reconstructed fields from the perilesional hippocampus representing the three zones and showing overall synaptic density (left) and synaptic density within Iba1+ cells only (right). Red, SV-2; green, Iba1; magenta, NeuN. B, Signal intensity analysis of synapses (SV-2) and microglia (Iba1) showing signal intensity correlates of each zone in $\boldsymbol{A}$. Gray shaded zone shown at bottom of histogram on each panel represent background signal. $C$, Heatmap of overall signal intensity of synapses and microglia across lesional and perilesional brain to define transition between the different zones. $\boldsymbol{D}, \boldsymbol{E}$, Representative examples of zone distribution in t-PA and B4Crry $+\mathrm{t}-\mathrm{PA}$-treated mice. Scale bars $=$ $20 \mu \mathrm{m} . \boldsymbol{F}$, Representative fields of zone 2 in the three different treatment groups. $\boldsymbol{G}$, Differences in areas of zones 3 and 2 quantified from $2 \mathrm{D}$-fields from the three different groups, and the density of synaptic material in microglia within zone 2 across the three different groups. ANOVA with Bonferroni. All pairwise comparisons were performed, and only significant results are highlighted with *. The difference in overall microglial density between 15 and $30 \mathrm{~d}$ is shown in Extended Data Figure 6-1. ns, not significant. 
A

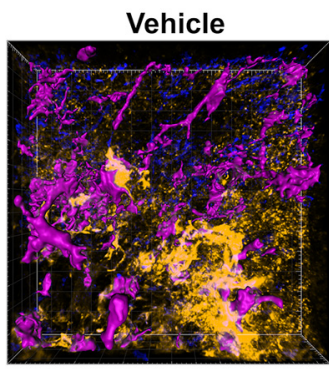

$\overline{20} \mu \mathrm{m}$

B

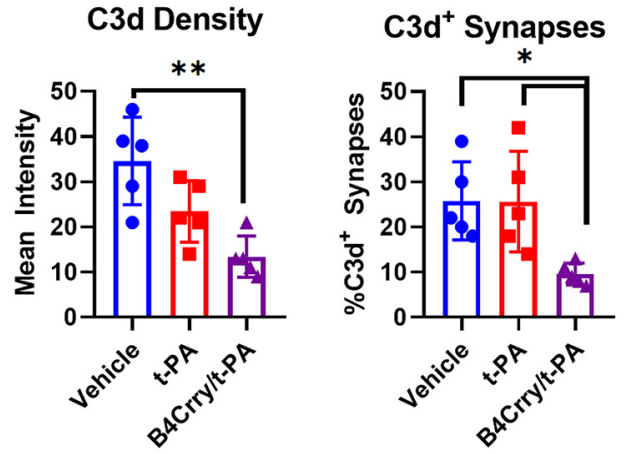

Iba1 | SV2 | C3d
Figure 7. B4Crry inhibited long-term complement C $3 d$ deposition on perilesional synapses. $A$, IF staining for C $3 d$ deposition in synapses within zone 2 of each of the three groups. Iba1, magenta; SV-2, blue; C3d, yellow. B, Quantification of $\mathrm{C} 3 \mathrm{~d}$ deposition and number of $\mathrm{C}^{+} \mathrm{d}^{+}$synapses in high-power fields from the different treatment groups (blue, vehicle; red, t-PA; magenta, B4(rry+t-PA). ANOVA with Bonferroni. $N=5 /$ group; ${ }^{*} p<0.05,{ }^{* *} p<0.01$. Bars $=$ mean \pm SEM. All pairwise comparisons were performed, and only significant results are highlighted with*
Table 1. Demographics and outcomes for patients undergoing thrombolysis or thrombectomy

\begin{tabular}{|c|c|c|c|c|}
\hline \multirow[b]{2}{*}{ Variable } & \multicolumn{2}{|c|}{ Thrombolysis group } & \multicolumn{2}{|c|}{ Thrombectomy } \\
\hline & $N$ & $\begin{array}{l}\text { Mean (SD) } \\
\text { Median [IQR] } \\
N(\%)\end{array}$ & $N$ & $\begin{array}{l}\text { Mean (SD) } \\
\text { Median [IQR] } \\
\mathrm{N}(\%)\end{array}$ \\
\hline Age (years) & 70 & $67(12)$ & 128 & 68 (14) \\
\hline Female gender & 70 & $36(51 \%)$ & 128 & $48(45 \%)$ \\
\hline White race & 70 & $41(59 \%)$ & 128 & $68(64 \%)$ \\
\hline \multicolumn{5}{|l|}{ Comorbidities } \\
\hline Diabetes & 70 & $28(40 \%)$ & 128 & $34(32 \%)$ \\
\hline Hypertension & 70 & $39(56 \%)$ & 128 & $51(48 \%)$ \\
\hline Atrial fibrillation & 70 & $52(74 \%)$ & 128 & $75(70 \%)$ \\
\hline Hyperlipidemia & 70 & $31(44 \%)$ & 128 & $35(33 \%)$ \\
\hline Prestroke mRS score & 70 & 0 [1] & 128 & $0[1]$ \\
\hline Admission NIHSS & 70 & $16(8)$ & 128 & $17(6)$ \\
\hline ASPECT score & 70 & $7(3)$ & 128 & $8(2)$ \\
\hline Anterior circulation & 70 & $65(93 \%)$ & 128 & $121(94 \%)$ \\
\hline ICA & & $23(33 \%)$ & $43(33 \%)$ & \\
\hline MCA & & $30(43 \%)$ & $59(46 \%)$ & \\
\hline Anterior cerebral artery & & $12(17 \%)$ & $19(15 \%)$ & \\
\hline Basilar artery & & $5(7 \%)$ & $7(6 \%)$ & \\
\hline Onset to presentation (min) & 70 & $168(69)$ & 128 & $432(589)$ \\
\hline Onset to reperfusion (min) & NA & NA & 128 & $459(591)$ \\
\hline Final TICl score & & & 128 & \\
\hline $0-2 A$ & NA & NA & & $8(7 \%)$ \\
\hline $2 B-3$ & NA & NA & & $99(93 \%)$ \\
\hline 90-d mRS score & 70 & $3[3]$ & 128 & $3[2]$ \\
\hline mRS $0-2$ & 70 & $28(40 \%)$ & 128 & $36(34 \%)$ \\
\hline mRS 3-6 & 70 & $42(60 \%)$ & 128 & $71(66 \%)$ \\
\hline
\end{tabular}

NA, not applicable.

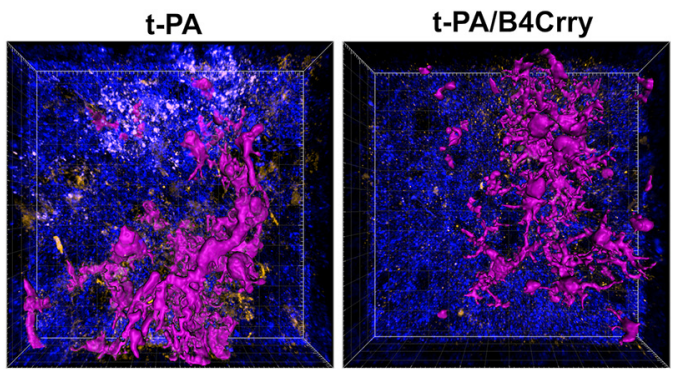

groups had higher and similar synaptic density in cortical sections compared with the vehicle group, but synaptic density in the hippocampus was significantly higher in $\mathrm{B} 4 \mathrm{Crry}+\mathrm{t}-\mathrm{PA}$-treated animals compared with both t-PA and vehicle-treated animals. There was no difference in hippocampal synaptic density between t-PA and vehicle (Fig. $5 A, B)$. Inversely correlated with synaptic density, there was higher $\mathrm{Iba}^{+}$cell density in the perilesional hippocampus of t-PA and vehicle-treated mice compared with B4Crry+ t-PA (Fig. 5B). However, despite lower overall hippocampal synaptic density in t-PA versus $\mathrm{t}-\mathrm{PA}+\mathrm{B} 4 \mathrm{Crry}$-treated animals, reconstruction of synaptic material and microglia/macrophages using Imaris revealed a higher density of synaptic material inside hippocampal microglia/macrophages in t-PA versus B4Crry+ t-PA-treated mice (Fig. 5C-E). Synaptic material within microglia was identified by masking all synaptic spots that do not fall within the reconstructed microglial surface in $3 \mathrm{D}$ space (Fig. 5C).

To better characterize this observation, we analyzed synaptic density, both inside and outside of microglia/macrophages over seven serial immunofluorescent fields within the same brain section, starting from the outside edge of the microgliotic scar (defined by confluent density of $\mathrm{Iba}^{+}$cells; Extended Data Fig. 5-1A). Moving away from the edge of the microgliotic scar, there was an expected drop in $\mathrm{Iba}^{+}$ cells and an increase in overall synaptic density in all treatment groups. However, in vehicle-treated animals, there was a notable spatially transient increase in synaptic material inside microglia/ macrophages between fields 2 and 5, peaking in field 4 (Extended Data Fig. 5-1B-D). A similar pattern for intracellular synaptic material was observed in t-PA-treated animals (Extended Data Fig. 5-1B,D). This region, denoted by the orange bar at the top of each panel in Extended Data Fig. 6-1,B-D represents an area adjacent to the microgliotic scar with a higher density of $\mathrm{Ibal}^{+}$cells compared with distant brain, with a higher frequency of synaptic material inside microglia/macrophages, but lower overall synaptic density; a definition for an area of active microglial elimination of synapses. In animals treated with $\mathrm{B} 4 \mathrm{Crry}+\mathrm{t}-\mathrm{PA}$, this region is limited to one to two fields abutting the scar, and there is a lower overall synaptic density within microglia compared with that observed with t-PA and vehicle (Extended Data Fig. 5-1B-D). Quantification of the extent of synaptic engulfment by microglia at the peak within this zone showed $\sim 60 \%$ reduction in animals treated with B4Crry+t-PA compared with t-PA or vehicle (Extended Data Fig. 5-1B-D). Similarly, the overall average density of synaptic material inside microglia across all fields was $\sim 60 \%$ less in animals treated with B4Crry+t-PA versus t-PA (Extended Data Fig. 5-1B-D). Notably, there was no apparent difference in neuronal cell density between B4Crry+t-PA and t-PA (Extended Data Fig. 5-1 $B-D)$. Thus, although t-PA and B4Crry $+\mathrm{t}-\mathrm{PA}$ treatment results in a similar reduction in infarct volume, only t-PA reperfused mice co-treated with B4Crry have reduced microgliosis and microglial engulfment of synapses, an event that otherwise continues chronically after stroke. 
The mean synaptic density in the hippocampus correlated significantly with retention of spatial memory on Barnes maze $\left(R^{2}\right.$ $=0.7, p<0.0001$; Fig. $5 F$ ), and with the slope of learning of spatial cues during training $\left(R^{2}=0.5, p<0.001\right.$; Fig. $\left.4 G\right)$.

Based on these findings, we classified the perilesional area into three zones on day 15 after MicroE stroke. Zone 1 represents an area of baseline synaptic density comparable to the contralateral hemisphere, with occasional $\mathrm{Iba}^{+}$cells (less than three cells per field). Zone 2 represents an area with 20 $60 \%$ loss of synaptic density, with increasing microglial activity and increased density of SV-2 particles within microglia, similar to Extended Data Fig. 5-1B-D. Zone 3 represents an area with loss of synaptic density and microgliotic scar formation (Fig. 6A). The area of ongoing microgliosis (zone 2) was quantified for each treatment group by reconstructing the histogram of synaptic (SV-2) signal intensity and microglial (Iba1) signal intensity with correlating heatmap showing the spatial change in signal intensity for microglia and synapses in different spatial zones (Fig. 6B-E). Treatment with t-PA or $\mathrm{B} 4 \mathrm{Crry}+\mathrm{t}-\mathrm{PA}$ significantly reduced the overall area of zone 3, whereas only B4Crry+t-PA significantly reduced the area of zone 2 compared with vehicle (Fig. $6 F, G$ ). Animals treated with $\mathrm{B} 4 \mathrm{Crry}+\mathrm{t}-\mathrm{PA}$ had significantly lower synaptic localization to microglia/macrophages within zone 2 compared with t-PA alone (Fig. 6F,G). These findings demonstrate that whereas treatment with t-PA alone reduces infarct size and areas of total tissue loss after stroke (zone 3), it does not inhibit ongoing microgliosis and associated loss of synaptic density in the ipsilesional brain (zone 2). This process is interrupted with acute inhibition of complement activation by B4Crry. Supporting the finding of ongoing microgliosis despite t-PA treatment, comparison of $\mathrm{Ibal}^{+}$cell density between 15 and $30 \mathrm{~d}$ after MicroE stroke showed a significant increase in vehicle and $\mathrm{t}$ PA-treated animals, but not in B4Crry $+\mathrm{t}-\mathrm{PA}$-treated mice (Extended Data Fig. 6-1).

To confirm a role for complement in elimination of perilesional synapses after stroke, we demonstrated robust deposition of C3d in zone 2 of vehicle-treated animals, which was inhibited with B4Crry+t-PA treatment, but not with t-PA alone (Fig. 7A, $B)$. Similarly, the percentage of synaptic particles positive for C3d was comparable in vehicle and t-PA-treated animals, and significantly lower in B4Crry+t-PA-treated mice (Fig. 7A,B).

\section{Cognitive and motor recovery following reperfusion therapy in human patients}

We next compared the difference in motor and cognitive recovery in human subjects receiving reperfusion therapy (IV-tPA or thrombectomy) after stroke using the Cog- 4 component and the motor components of the NIHSS. Based on selection criteria described in Materials and Methods, of 195 patients treated with t-PA and 561 patients treated with endovascular thrombectomy (ET), 70 t-PA-treated and 128 ET successfully-treated patients were included in this study (Table 1). Although improvement over $90 \mathrm{~d}$ was observed in both domains, patients receiving reperfusion therapy (IV-tPA or ET) showed a significantly more pronounced improvement in motor compared with cognitive deficits $90 \mathrm{~d}$ after stroke (Fig. 8A,B). We then compared patients undergoing
ET with successful recanalization (Recan, $N=150$ ) defined as TICI score (2B-3) to patients without successful recanalization (No Recan, $N=50$; no reperfusion) to assess the impact of recanalization on recovery (Table 2). Patients within the Recan and No Recan groups were matched by age, gender, location, admission NIHSS, and onset-to-groin time (Table 2). Although successful recanalization was associated with a significant improvement in 90-d mRS and the motor components of the NIHSS, there was no significant improvement on the Cog- 4 cognitive component of the NIHSS at $90 \mathrm{~d}$ (Table 2). There was no significant difference between the two groups on short-term memory retention assessed by the three-object recall test one week after stroke (Table 2), and there was no significant difference in scores on MoCA testing between patients who underwent the test from each group (Table 2). These findings support our preclinical observations that restoration of blood supply to the brain may not be sufficient for optimal cognitive recovery.

\section{Discussion}

This work introduces a novel role for complement in stroke synaptic degeneration after stroke, a process that starts acutely and persists chronically independent of cerebral revascularization. Complement activation and opsonization directs microglia-dependent phagocytosis of synapses for at least $30 \mathrm{~d}$ poststroke and limits cognitive recovery in mice. Our study investigated neuroinflammation independent of infarct volume using a clinically relevant paradigm that involves t-PA and/or complement inhibitor-treated mice. Complement inhibition improved the safety, efficacy and treatment window of reperfusion therapy, and limited hemorrhagic transformation. This work is first to show that stroke reperfusion does not prevent cognitive decline in mice; however, adjuvant or isolated complement inhibition improved cognitive performance despite comparable infarct volume. We show that the therapeutic effect size of pharmacological or mechanical reperfusion, in mice or humans is significantly lower for cognitive compared with motor outcomes. The mechanism of action of B4Crry, the targeted complement inhibitor used in this study, is shown in Figure 9 and has been established in prior work (Atkinson et al., 2015; Alawieh and Tomlinson, 2016; Alawieh et al., 2018b).

Reperfusion therapy with intravenous t-PA or thrombectomy is the current standard of care in IS, and eligibility is dependent on the timing of presentation and risk of hemorrhagic conversion (Powers et al., 2019). The efficacy of stroke thrombectomy was shown in several clinical trials (Berkhemer et al., 2015; Campbell et al., 2015; Jovin et al., 2015, 2018; Saver et al., 2015; 
Table 2. Demographics and outcomes for patients treated with MT dichotomized into whether recanalization was achieved

\begin{tabular}{|c|c|c|c|c|c|c|}
\hline \multirow[b]{2}{*}{ Variable } & \multicolumn{2}{|c|}{ Non-recanalized TICI 0-2A } & \multicolumn{2}{|c|}{ Successfully recanalized (TICI 2B+) } & \multirow[b]{2}{*}{ Test } & \multirow[b]{2}{*}{$p$ value } \\
\hline & N & $\begin{array}{l}\text { Mean (SD) } \\
\text { Median [IQR] } \\
N(\%)\end{array}$ & N & $\begin{array}{l}\text { Mean (SD) } \\
\text { Median [IQR] } \\
N(\%)\end{array}$ & & \\
\hline Age (years) & 50 & $65(15)$ & 150 & $66(14)$ & $t$ test & 0.437 \\
\hline Female gender & 50 & $23(46 \%)$ & 150 & $64(43 \%)$ & $\chi^{2}$ & 0.68 \\
\hline White race & 50 & & 150 & & $\chi^{2}$ & \\
\hline Hypertension & 50 & & 150 & & $\chi^{2}$ & \\
\hline Atrial fibrillation & 50 & & 150 & & $\chi^{2}$ & \\
\hline Hyperlipidemia & 50 & & 150 & & $x^{2}$ & \\
\hline Prestroke mRS score & 50 & $0[1]$ & 150 & $0[1]$ & MW & 0.897 \\
\hline Admission NIHSS & 50 & $15.5(7)$ & 150 & $17(7)$ & $t$ test & 0.474 \\
\hline ASPECT score & 50 & $8(2)$ & 150 & $8(2)$ & $t$ test & 0.989 \\
\hline Basilar artery & & $3(6 \%)$ & & $7(5 \%)$ & & \\
\hline Onset to groin (min) & 50 & $491(371)$ & 150 & $401(527)$ & $t$ test & 0.751 \\
\hline Onset to recanalization (min) & NA & NA & 150 & & & \\
\hline Final TICI score & 50 & & 150 & & NA & NA \\
\hline $0-2 A$ & & $50(100 \%)$ & & $0(0.0 \%)$ & & \\
\hline $2 B-3$ & & $0(0.0 \%)$ & & $150(100 \%)$ & & \\
\hline 90-d mRS score & 50 & $4[3]$ & 150 & $3[3]$ & MW & 0.0003 \\
\hline mRS $0-2$ & 50 & $15(30 \%)$ & 150 & $72(48 \%)$ & $\chi^{2}$ & 0.026 \\
\hline mRS 3-6 & 50 & $35(70 \%)$ & 150 & $78(52 \%)$ & $\chi^{2}$ & 0.026 \\
\hline \multicolumn{7}{|l|}{ NIHSS improvement 90 vs admission } \\
\hline \% Improvement in NIHSS, motor & 50 & $50 \%(49 \%)$ & 150 & $72 \%(42 \%)$ & $t$ test & 0.001 \\
\hline \% Improvement in NIHSS, Cog-4 & 50 & $60 \%(41 \%)$ & 150 & $70 \%(38 \%)$ & $t$ test & 0.07 \\
\hline$\%$ Objects recalled (short-term memory)* & 44 & $21 \%(38 \%)$ & 144 & $26 \%(39 \%)$ & $t$ test & 0.545 \\
\hline
\end{tabular}

NA, not applicable; MW, Mann-Whitney test; MoCA, Montreal cognitive assessment; ${ }^{*}$, patients assessed for performance on three-object recall test at one to three weeks after stroke. Percentage of object recalled is reported; **, MoCA test was performed on subset of patients between 60 and $90 \mathrm{~d}$ after stroke.

Bracard et al., 2016; Albers et al., 2018); yet, there was consistent mismatch between the rate of vessel recanalization(reperfusion) and good functional outcome (Fig. 10). Around 50\% of treated cohorts failed to achieve functional independence at $90 \mathrm{~d}$, and only $50 \%$ of patients showed improvement in deficit scores within $24 \mathrm{~h}$ (Goyal et al., 2016). Both t-PA and ET are also associated with the risk of ICH and reperfusion injury, and older patients and patients with posterior circulation stroke are at increased risk (Alawieh et al., 2018d, 2019). These limitations of reperfusion therapy hold true for ideal candidates at highly skilled centers, and prove that penumbral dysfunction persists despite successful reperfusion in a large patient subset. Thus, closing the gap between reperfusion success and functional recovery and increasing the window of therapeutic eligibility is likely to involve targeting alternative pathophysiological mechanisms such as poststroke neuroinflammation.

We show that reperfusion is associated with increased risk of BBB dysfunction, and ICH supporting data from human studies (Kase et al., 2001; Berkhemer et al., 2015; Campbell et al., 2015; Jovin et al., 2015, 2018; Saver et al., 2015; Bracard et al., 2016; Alawieh et al., 2018d; Albers et al., 2018). Data from these studies report around 5\% - 15\% risk of ICH after t-PA and a 5\% risk after thrombectomy, even in highly selected patient populations with increased risk after delayed treatment. These findings were replicated in our murine studies where delayed reperfusion with t-PA ( 4 or $6 \mathrm{~h}$ after MicroE) increased the risk of acute mortality and hemorrhage and resulted in worsened neurologic deficits. Reperfusion was also associated with increased complement activation in the ipsilateral hemisphere, and that complement activation is increased when reperfusion is delayed. Prior work in mechanically reperfused animals (tMCAO) additionally treated with t-PA, showed an increase in complement activation with $\mathrm{t}$ $\mathrm{PA}$ treatment suggesting a role of t-PA in direct activation of complement via an extrinsic pathway (Zhao et al., 2017). They show that t-PA resulted in an increase in cerebral C3a release directly correlated with the extent of hemorrhage and edema. The role of t-PA in promoting hemorrhage after stroke and reperfusion is consistent with our findings. However, we show that inhibition of C3-convertase locally by B4Crry inhibited $\mathrm{C} 3$ activation compared with t-PA alone. Thus, although t-PA may activate complement independent of $\mathrm{C} 3$ convertase via the extrinsic pathway, t-PA-driven $\mathrm{C} 3$ activation is more likely to be secondary to reperfusion-associated complement activation that leads to assembly of C3 convertase. In addition to the reduction in t-PA associated complement activation, hemorrhage and acute mortality after MicroE stroke, both motor and cognitive recovery were quantitatively higher with combination therapy (B4Crry $+\mathrm{t}$-PA) compared with either therapy alone, indicating an additive effect. Therefore, inhibition of complement-induced inflammation after reperfusion therapy is likely to improve the safety and efficacy profile of thrombolytic therapy, and may potentially increase the number of stroke patients eligible for treatment. 


\section{Pre-ischemia Status}

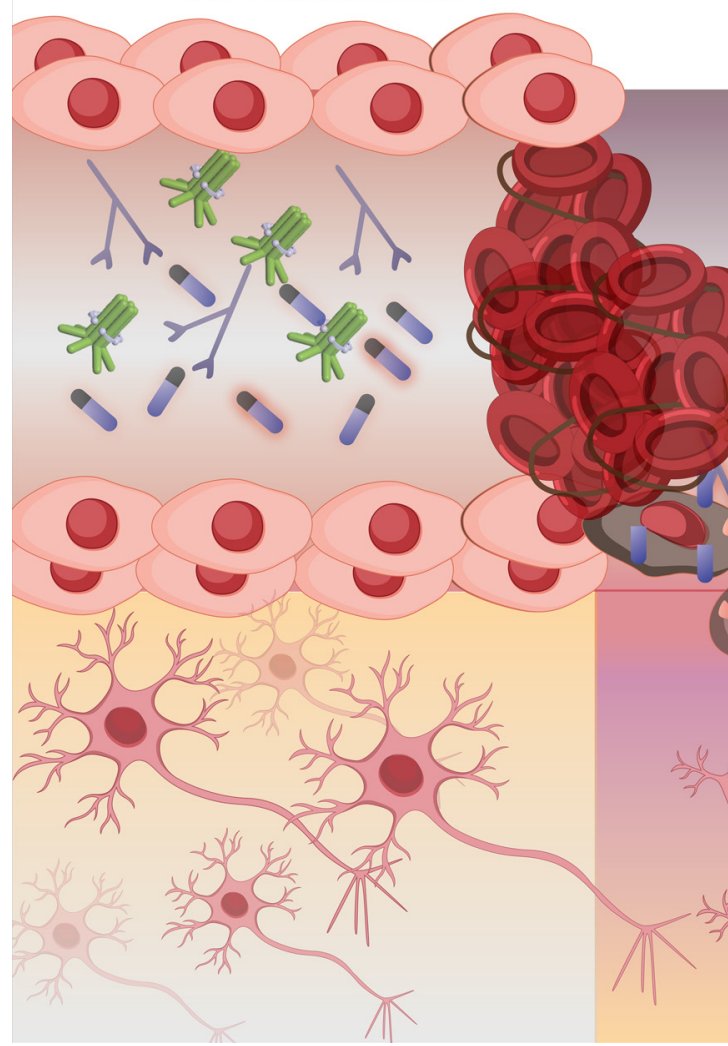
Post-ischemia Response
No Inhibitor

\section{Post-ischemia Response [+B4Crry]}

Figure 9. Illustration of mechanism of action of B4Crry. Left panel, In the normal brain, endothelial cells, neurons and other parenchymal cells do not express stress-related antigens. Middle panel, Following ischemic insult, the expression of neoepitopes (or danger associated molecular patterns, DAMPs) is induced on the surface of these cells. One such neoepitope expressed in the postischemic murine brain is a post-translational modification of Annexin IV, which is recognized by the B4scFv targeting moiety used in this study. In the absence of complement inhibition, this neoepitope (and others) engages circulating natural IgM antibodies and activate complement, leading to the assembly of the $C 3$ convertase and subsequent generation of $C 3 \mathrm{~b}$ opsonins and C3a anaphylatoxin. Right panel, The fusion protein B4Crry binds specifically to sites of B4-DAMP expression, thus competing with lgM binding of B4 specificity, while simultaneously inhibiting the activity of the $\mathrm{C} 3$ convertase via its Crry component. This ultimately leads to the interruption of the neuroinflammatory cascade that is otherwise activated on endothelial cells and neurons.

\section{Mismatch Between Revascularization Rates and Rates of Good Outcomes after Stroke Thrombectomy}

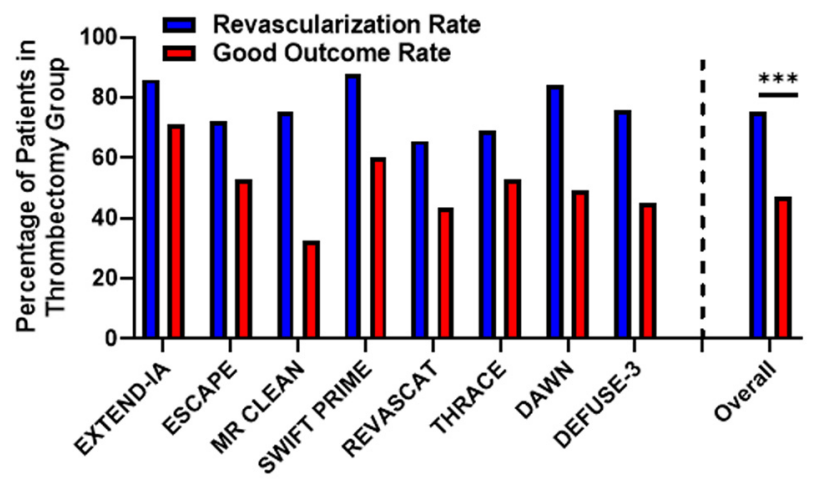

\section{Randomized Trials}

Figure 10. Mismatch between rates of successful revascularization (define as mTICI $2 B$ score or higher) and rates of good functional recovery (mRS $0-2$ ) after stroke thrombectomy in major trials. Data pooled from all trials $(N=988)$ was summarized and comparison was performed using the Fisher exact test; ${ }^{* *} p<0.001$.

As noted in the introduction, B4Crry inhibits all complement activation products with effector function that are generated subsequent to C3 cleavage (C3 opsonins, C3a, C5a, and C5b-9). In this regard, it is noteworthy that that $\mathrm{C} 3 \mathrm{a}$ and $\mathrm{C} 5 \mathrm{a}$ have been shown to have neuroprotective properties, and to promote neural plasticity and regeneration (for review, see Alawieh et al., 2015a, 2018a). However, this is not in apparent conflict with the current data, since $\mathrm{C} 3 \mathrm{a}$ and $\mathrm{C} 5 \mathrm{a}$ appear to exert their protective effects in the subacute or chronic phase after CNS injury. In the current work, complement inhibition (and thus inhibition of C3a and C5a generation) was applied only acutely, at which time complement has a clearly defined pathogenic role in stroke (Alawieh et al., 2015b, 2018c).

Beyond acute outcomes, chronic neuroinflammation has been associated with poststroke cognitive decline (Barrett et al., 2011; Smith et al., 2015; Kalaria et al., 2016; Elkins et al., 2017). However, cognitive outcomes were not assessed in the major randomized controlled trials which used the mRS of disability as primary outcome (Kase et al., 2001; Berkhemer et al., 2015; Campbell et al., 2015; Jovin et al., 2015, 2018; Saver et al., 2015; Bracard et al., 2016; Alawieh et al., 2018d; Albers et al., 2018). In a systematic review of thrombolytic therapy studies, thrombolysis did not have a significant effect on long-term cognitive outcomes including global cognition, language, and memory domains (Broome et al., 2016). Our data suggests that for stroke patients presenting with both cognitive and motor deficits, a more prominent recovery in motor outcomes compared with cognition is observed following reperfusion. These findings, although limited in terms of being retrospective in nature, with a lack of standardized neuropsychological testing, still provide evidence to support our preclinical investigation of limited cognitive recovery after reperfusion. 
Using our MicroE model, we demonstrated that although early thrombolysis reduced infarct volume and improved motor performance, cognitive performance was indistinguishable from controls during both learning and retention phases of spatial learning and passive avoidance tasks. In our paradigms, cognitive performance was improved with B4Crry treatment, but not t-PA therapy. We also demonstrate that supplementing thrombolytic therapy with EE exposure, used as a preclinical model of rehabilitation, does not bridge the gap in cognitive recovery compared with B4Crry treatment. Specifically, t-PA therapy alone did not improve cognitive recovery after MicroE stroke, and did not prime the reperfused brain for a better cognitive response to rehabilitation and a similar response to rehabilitation was seen in comparison to vehicle counterparts. Cognitive performance may be improved by neuroregeneration and migration of neuronal progenitor cells to the ischemic brain, but the absence of differences in $\mathrm{Dcx}^{+}$cell density between t-PA and B4Crry-treated groups indicate that the difference in the pattern of cognitive recovery between the two treatments is not due to increased neurogenesis. These data are consistent with the impact of B4Crry on delayed cognitive recovery being dependent on inhibition of a complement-exacerbated neuroinflammation.

Complement-dependent microglial activation after stroke was previously associated with loss of live neurons tagged by complement opsonins for destruction (Alawieh et al., 2018b). Here, we did not observe a difference in neuronal density in the cortex or hippocampus between t-PA and B4Crry+t-PA treatment groups at $30 \mathrm{~d}$. Thus, the loss of neurons is unlikely to explain the long-term pathology behind poor cognitive performance in t-PA-treated animals compared with B4Crry+t-PA. Prior work using models of neurodegenerative disorders demonstrated that loss of synapses is an early predictor of cognitive decline (Terry et al., 1991; Stephan et al., 2012; Hong et al., 2016; Vasek et al., 2016). We therefore explored whether the cognitive decline observed in our chronic stroke model may be due to complement-dependent loss of synapses. We found that $30 \mathrm{~d}$ after stroke and successful recanalization, active microgliosis was associated with decreased synaptic density in the ipsilesional hippocampus, and that loss of synaptic material correlated with the pattern of cognitive recovery after stroke. B4Crry inhibited synaptic phagocytosis and preserved synaptic density in the ipsilateral hippocampus, allowing for optimal cognitive recovery. Synaptic degeneration is a known pathophysiological finding in neurodegenerative diseases including Alzheimer's disease or glaucoma (Terry et al., 1991; Stephan et al., 2012; Overk and Masliah, 2014; Hong et al., 2016; Vasek et al., 2016; Williams et al., 2016), and our findings demonstrate that it is an ongoing long-term event after IS that may underlie chronic deterioration in cognitive performance and may predispose to poststroke dementia.

Although synaptic loss precedes neuronal loss in neurodegenerative disease, we did not see a difference in neuronal density between t-PA and B4Crry+t-PA-treated animals. This could be due to the acute nature of brain injury in stroke compared with the chronicity of neurodegenerative disease, and it is possible that a change in synaptic density may be followed by loss of neuronal density at several months after stroke. It is not clear why the loss of synapses was more robust in the hippocampus compared with ipsilesional cortex after stroke and t-PA treatment. A potential explanation is the difference in vascular density and vascular patterns between the different regions. The area with major differences between t-PA and B4Crry+t-PA-treated animals appears to be limited to the CA1 region of the hippocampus, where microgliosis zones 2 and 3 occurred (Fig. 6). The CA1 region of the hippocampus has lower capillary density and a higher risk of BBB leakage after ischemic and excitotoxic injury compared with the cortex which may explain this pattern (Cavaglia et al., 2001). Despite the fact that the hippocampal area in mice is predominantly supplied by the PCA in mice, the involvement of the hippocampus in ischemia-reperfusion injury in this model is common since poststroke secondary injury, including the inflammatory response, cytotoxic edema and reactive vasoconstriction, expands within the brain via spatial proximity and does not specifically respect vascular territories (Chen et al., 2015; Alawieh et al., 2018a). Inhibition of complementdriven neuroinflammation provided an additive effect to t-PA by inhibiting this secondary progression of injury beyond the initial core region.

Prior studies suggested that t-PA proteolytic activity may alter synaptic signaling (for review, see Samson and Medcalf, 2006). However, due to its short half-life and single acute administration in our study, it is unlikely that the difference in pattern of synaptic loss and cognitive dysfunction is related to intrinsic properties of t-PA, or to its proposed role in synaptic signaling. To further support this conclusion, complement inhibition reverses cognitive dysfunction in our model without interfering with the proteolytic activity of t-PA.

\section{References}

Alawieh A, Tomlinson S (2016) Injury site-specific targeting of complement inhibitors for treating stroke. Immunol Rev 274:270-280.

Alawieh A, Elvington A, Tomlinson S (2015a) Complement in the homeostatic and ischemic brain. Front Immunol 6:417.

Alawieh A, Elvington A, Zhu H, Yu J, Kindy MS, Atkinson C, Tomlinson S (2015b) Modulation of post-stroke degenerative and regenerative processes and subacute protection by site-targeted inhibition of the alternative pathway of complement. J Neuroinflammation 12:247.

Alawieh A, Langley F, Tomlinson S (2018a) The inflammatory response and its effect on rehabilitation-induced repair processes after stroke. In: Cellular and molecular approaches to regeneration and repair, pp 509520. New York: Springer.

Alawieh A, Langley EF, Tomlinson S (2018b) Targeted complement inhibition salvages stressed neurons and inhibits neuroinflammation after stroke in mice. Sci Transl Med 10:eaao6459.

Alawieh A, Andersen M, Adkins DL, Tomlinson S (2018c) Acute complement inhibition potentiates neurorehabilitation and enhances tPA-mediated neuroprotection. J Neurosci 38:6527-6545.

Alawieh A, Pierce AK, Vargas J, Turk AS, Turner RD, Chaudry MI, Spiotta AM (2018d) The golden 35 min of stroke intervention with ADAPT: effect of thrombectomy procedural time in acute ischemic stroke on outcome. J Neurointervent Surg 10:213-220.

Alawieh A, Starke RM, Chatterjee AR Turk A, De Leacy R, Rai AT Fargen K, Kan P, Singh J, Vilella L, Nascimento FA Dumont TM McCarthy D Spiotta AM (2019) Outcomes of endovascular thrombectomy in the elderly: a 'real-world' multicenter study. J Neurointerv Surg 11:545-553.

Alawieh A, Wang X, Narang A, Tomlinson S (2016) Thromboembolic model of cerebral ischemia and reperfusion in mice. In: Injury Models of the central nervous system: Methods and protocols 1st Edition (Kobeissy F, Dixon CE, Hayes R, Mondello S, eds). Totowa: Humana Press.

Albers GW, Marks MP, Kemp S, Christensen S, Tsai JP, Ortega-Gutierrez S, McTaggart RA, Torbey MT, Kim-Tenser M, Leslie-Mazwi T, Sarraj A, Kasner SE, Ansari SA, Yeatts SD, Hamilton S, Mlynash M, Heit JJ, Zaharchuk G, Kim S, Carrozzella J, et al. (2018) Thrombectomy for stroke at 6 to 16 hours with selection by perfusion imaging. N Engl J Med 378:708-718.

Allred RP, Adkins DL, Woodlee MT, Husbands LC, Maldonado MA, Kane JR, Schallert T, Jones TA (2008) The vermicelli handling test: a simple quantitative measure of dexterous forepaw function in rats. J Neurosci Methods 170:229-244.

Atkinson C, Qiao F, Yang X, Zhu P, Reaves N, Kulik L, Goddard M, Holers VM, Tomlinson S (2015) Targeting pathogenic postischemic self-recognition by natural IgM to protect against posttransplantation cardiac reperfusion injury. Circulation 131:1171-1180. 
Babadjouni RM, Walcott BP, Liu Q, Tenser MS, Amar AP, Mack WJ (2017) Neuroprotective delivery platforms as an adjunct to mechanical thrombectomy. Neurosurg Focus 42:E4.

Barrett KM, Brott TG, Brown RD Jr, Carter RE, Geske JR, Graff-Radford NR, McNeil RB, Meschia JF; Mayo Acute Stroke Trial for Enhancing Recovery (MASTER) Study Group (2011) Enhancing recovery after acute ischemic stroke with donepezil as an adjuvant therapy to standard medical care: results of a phase IIA clinical trial. J Stroke Cerebrovasc Dis 20:177-182.

Berkhemer OA, Fransen PSS, Beumer D, van den Berg LA, Lingsma HF, Yoo AJ, Schonewille WJ, Vos JA, Nederkoorn PJ, Wermer MJH, van Walderveen MAA, Staals J, Hofmeijer J, van Oostayen JA, Lycklama à Nijeholt GJ, Boiten J, Brouwer PA, Emmer BJ, de Bruijn SF, van Dijk LC, et al. (2015) A randomized trial of intraarterial treatment for acute ischemic stroke. N Engl J Med 372:11-20.

Bracard S, Ducrocq X, Mas JL, Soudant M, Oppenheim C, Moulin T, Guillemin F, investigators T; THRACE Investigators (2016) Mechanical thrombectomy after intravenous alteplase versus alteplase alone after stroke (THRACE): a randomised controlled trial. Lancet Neurol 15:1138-1147.

Broome LJ, Battle CE, Lawrence M, Evans PA, Dennis MS (2016) Cognitive outcomes following thrombolysis in acute ischemic stroke: a systematic review. J Stroke Cerebrovasc Dis 25:2868-2875.

Campbell BCV, Mitchell PJ, Kleinig TJ, Dewey HM, Churilov L, Yassi N, Yan B, Dowling RJ, Parsons MW, Oxley TJ, Wu TY, Brooks M, Simpson MA, Miteff F, Levi CR, Krause M, Harrington TJ, Faulder KC, Steinfort BS, Priglinger M, et al. (2015) Endovascular therapy for ischemic stroke with perfusion-imaging selection. N Engl J Med 372:1009-1018.

Cavaglia M, Dombrowski SM, Drazba J, Vasanji A, Bokesch PM, Janigro D (2001) Regional variation in brain capillary density and vascular response to ischemia. Brain Res 910:81-93.

Chen C, Leys D, Esquenazi A (2013) The interaction between neuropsychological and motor deficits in patients after stroke. Neurology 80:S27-S34.

Chen Y, Zhu W, Zhang W, Libal N, Murphy SJ, Offner H, Alkayed NJ (2015) A novel mouse model of thromboembolic stroke. J Neurosci Methods 256:203-211.

Chiang T, Messing RO Chou WH (2011) Mouse model of middle cerebral artery occlusion. J Vis Exp. Advance online publication. Retrieved Feb 13, 2011. doi: $10.3791 / 2761$

Elkins J, Veltkamp R, Montaner J, Johnston SC, Singhal AB, Becker K, Lansberg MG, Tang W, Chang I, Muralidharan K, Gheuens S, Mehta L, Elkind MSV (2017) Safety and efficacy of natalizumab in patients with acute ischaemic stroke (ACTION): a randomised, placebo-controlled, double-blind phase 2 trial. Lancet Neurol 16:217-226.

Goyal M, Menon BK, van Zwam WH, Dippel DWJ, Mitchell PJ, Demchuk AM, Dávalos A, Majoie CBLM, van der Lugt A, de Miquel MA, Donnan GA, Roos YBWEM, Bonafe A, Jahan R, Diener HC, van den Berg LA, Levy EI, Berkhemer OA, Pereira VM, Rempel J, et al. (2016) Endovascular thrombectomy after large-vessel ischaemic stroke: a meta-analysis of individual patient data from five randomised trials. Lancet 387:1723-1731.

Hacke W, Kaste M, Bluhmki E, Brozman M, Dávalos A, Guidetti D, Larrue V, Lees KR, Medeghri Z, Machnig T, Schneider D, von Kummer R, Wahlgren N, Toni D, IE (2008) Thrombolysis with alteplase 3 to 4.5 hours after acute ischemic stroke. N Engl J Med 359:1317-1329.

Hajjar K, Fulton RL, Diener HC, Lees KR, Alexandrov A, Bath PMW, Bluhmki E, Claesson L, Curram J, Davis SM, Donnan G, Diener HC, Fisher M, Gregson B, Grotta J, Hacke W, Hennerici MG, Hommel M, Kaste M, Lees KR, et al. (2013) Does the cognitive measure Cog-4 show improvement among patients treated with thrombolysis after acute stroke? Int J Stroke 8:652-656.

Hong S, Beja-Glasser VF, Nfonoyim BM, Frouin A, Li S, Ramakrishnan S, Merry KM, Shi Q, Rosenthal A, Barres BA, Lemere CA, Selkoe DJ, Stevens B (2016) Complement and microglia mediate early synapse loss in Alzheimer mouse models. Science 352:712-716.

Iadecola C, Anrather J (2011) The immunology of stroke: from mechanisms to translation. Nat Med 17:796-808.

Jovin TG, Chamorro A, Cobo E, de Miquel MA, Molina CA, Rovira A, San Román L, Serena J, Abilleira S, Ribó M, Millán M, Urra X, Cardona P, López-Cancio E, Tomasello A, Castaño C, Blasco J, Aja L, Dorado L, Quesada H, et al. (2015) Thrombectomy within 8 hours after symptom onset in ischemic stroke. N Engl J Med 372:2296-2306.
Jovin TG, Nogueira RG, Investigators D; DAWN Investigators (2018) Thrombectomy 6 to 24 hours after stroke. N Engl J Med 378:1161-1162.

Kalaria RN, Akinyemi R, Ihara M (2016) Stroke injury, cognitive impairment and vascular dementia. Biochim Biophys Acta 1862:915-925.

Kase CS, Furlan AJ, Wechsler LR, Higashida RT, Rowley HA, Hart RG, Molinari GF, Frederick LS, Roberts HC, Gebel JM, Sila CA, Schulz GA, Roberts RS, Gent M (2001) Cerebral hemorrhage after intra-arterial thrombolysis for ischemic stroke: the PROACT II trial. Neurology 57:1603-1610.

Kulik L, Fleming SD, Moratz C, Reuter JW, Novikov A, Chen K, Andrews KA, Markaryan A, Quigg RJ, Silverman GJ, Tsokos GC, Holers VM (2009) Pathogenic natural antibodies recognizing annexin IV are required to develop intestinal ischemia-reperfusion injury. J Immunol 182:5363-5373.

Metz GA, Whishaw IQ (2009) The ladder rung walking task: a scoring system and its practical application. J Vis Exp. Advance online publication. Retrieved June 12, 2009. doi: 10.3791/1204.

Overk CR, Masliah E (2014) Pathogenesis of synaptic degeneration in Alzheimer's disease and Lewy body disease. Biochem Pharmacol 88:508516.

Powers WJ, Rabinstein AA, Ackerson T, Adeoye OM, Bambakidis NC, Becker K, Biller J, Brown M, Demaerschalk BM, Hoh B, Jauch EC, Kidwell CS, Leslie-Mazwi TM, Ovbiagele B, Scott PA, Sheth KN, Southerland AM, Summers DV, Tirschwell D; American Heart Association Stroke Council (2019) 2018 Guidelines for the early management of patients with acute ischemic stroke: a guideline for healthcare professionals from the American Heart Association/American Stroke Association. Stroke 50:e46-e110.

Presumey J, Bialas AR, Carroll MC (2017) Complement system in neural synapse elimination in development and disease. Adv Immunol 135:53-79.

Samson AL, Medcalf RL (2006) Tissue-type plasminogen activator: a multifaceted modulator of neurotransmission and synaptic plasticity. Neuron 50:673-678.

Saver JL, Goyal M, Bonafe A, Diener HC, Levy EI, Pereira VM, Albers GW, Cognard C, Cohen DJ, Hacke W, Jansen O, Jovin TG, Mattle HP, Nogueira RG, Siddiqui AH, Yavagal DR, Baxter BW, Devlin TG, Lopes DK, Reddy VK, et al. (2015) Stent-retriever thrombectomy after intravenous t-PA vs. t-PA alone in stroke. N Engl J Med 372:2285-2295.

Smith CJ, Denes A, Tyrrell PJ, Di Napoli M (2015) Phase II anti-inflammatory and immune-modulating drugs for acute ischaemic stroke. Expert Opin Investig Drugs 24:623-643.

Stephan AH, Barres BA, Stevens B (2012) The complement system: an unexpected role in synaptic pruning during development and disease. Annu Rev Neurosci 35:369-389.

Terry RD, Masliah E, Salmon DP, Butters N, DeTeresa R, Hill R, Hansen LA, Katzman R (1991) Physical basis of cognitive alterations in Alzheimer's disease: synapse loss is the major correlate of cognitive impairment. Ann Neurol 30:572-580.

Turk AS, Frei D, Fiorella D, Mocco J, Baxter B, Siddiqui A, Spiotta A, Mokin M, Dewan M, Quarfordt S, Battenhouse H, Turner R, Chaudry I (2014) ADAPT FAST study: a direct aspiration first pass technique for acute stroke thrombectomy. J Neurointerv Surg 6:260-264.

Uyama O, Okamura N, Yanase M, Narita M, Kawabata K, Sugita M (1988) Quantitative evaluation of vascular permeability in the gerbil brain after transient ischemia using Evans blue fluorescence. J Cereb Blood Flow Metab 8:282-284.

Vasek MJ, Garber C, Dorsey D, Durrant DM, Bollman B, Soung A, Yu J, Perez-Torres C, Frouin A, Wilton DK, Funk K, DeMasters BK, Jiang X, Bowen JR, Mennerick S, Robinson JK, Garbow JR, Tyler KL, Suthar MS, Schmidt RE, et al. (2016) A complement-microglial axis drives synapse loss during virus-induced memory impairment. Nature 534:538-543.

Williams PA, Tribble JR, Pepper KW, Cross SD, Morgan BP, Morgan JE, John SW, Howell GR (2016) Inhibition of the classical pathway of the complement cascade prevents early dendritic and synaptic degeneration in glaucoma. Mol Neurodegener 11:26.

Zhang L, Schallert T, Zhang ZG, Jiang Q, Arniego P, Li Q, Lu M, Chopp M (2002) A test for detecting long-term sensorimotor dysfunction in the mouse after focal cerebral ischemia. J Neurosci Methods 117:207-214.

Zhao XJ, Larkin TM, Lauver MA, Ahmad S, Ducruet AF (2017) Tissue plasminogen activator mediates deleterious complement cascade activation in stroke. PLoS One 12:e0180822. 